\title{
Investigations into a plankton population model: Mortality and its importance in climate change scenarios
}

Roger Cropp ${ }^{a, *}$, John Norbury ${ }^{b}$

a Faculty of Environmental Sciences, Griffith University, Nathan, Queensland, Australia

b Mathematical Institute, University of Oxford, 24-29 St. Giles, Oxford OX3 9DU, UK

\section{A R T I C L E I N F O \\ Article history: \\ Received 26 August 2005 \\ Received in revised form \\ 21 July 2006 \\ Accepted 5 September 2006}

Keywords:

Plankton model

Dimethylsulphide

Climate change

\begin{abstract}
A B S T R A C T
The potential for marine plankton ecosystems to influence climate by the production of dimethylsulphide (DMS) has been an important topic of recent research into climate change. Several General Circulation Models, used to predict climate change, have or are being modified to include interactions of ecosystems with climate. Climate change necessitates that parameters within ecosystem models must change during long-term simulations, especially mortality parameters that increase as organisms are pushed toward the boundaries of their thermal tolerance. Changing mortality parameters can have profound influences on ecosystem model dynamics. There is therefore a pressing need to understand the influence of varying mortality parameters on the long-term behaviour of ecosystem models. This work examines the sensitivity of a model of DMS production by marine ecosystems to variations in three linear mortality coefficients. Significant differences in behaviour are observed, and we note the importance of these results in formulating ecosystem models for application in simulations of climate change.
\end{abstract}

(c) 2006 Elsevier B.V. All rights reserved.

\section{Introduction}

\begin{abstract}
Climate models have investigated the greenhouse effect, the tendency of the atmosphere to alter its characteristics to sunlight so that different proportions of the Earth's black body radiation are trapped by the atmosphere, thus leading to different climates. The most important component of this greenhouse effect studied in the past decades has been carbon dioxide $\left(\mathrm{CO}_{2}\right)$ in the atmosphere. Its storage in forests and oceans, and its production by industry, are subjects of major interest. More recently dimethylsulphide (DMS) molecules in the atmosphere have been identified as a potentially significant modifier of solar radiation reflectance (Charlson et al., 1987). Hence the production and emission of this gas to the atmosphere from plankton blooms in the upper regions of the oceans has been a subject of growing interest, from observational, experimental and scientific modelling perspectives.
\end{abstract}

We consider the GMSK model introduced by Moloney et al. (1986) and subsequently developed by Gabric et al. (1993) to include dimethylsulphoniopropionate (DMSP) and DMS. This model considers a self-contained amount of biological matter (held in various forms expressed as dissolved inorganic nutrient $(N)$, phytoplankton $(P)$, bacteria $(B)$, zooflagellates $(F)$ and zooplankton $(Z)$ in a common currency of nitrogen concentration), and evaluates the amount of DMSP and DMS (expressed in a currency of sulphur concentration) produced over a plankton bloom cycle. The model consists of linked ordinary differential equations (ODEs) describing the changes in concentrations of the above quantities. This model has proved useful in reproducing spatial distributions and depth profiles of DMS observed in the ocean (Gabric et al., 1999; Cropp et al., 2004) and in predicting the potential for biogenic DMS to ameliorate climate change (Gabric et al., 2003, 2004). The work of Cropp et al. (2004) shows that understanding the

\footnotetext{
* Corresponding author.

E-mail address: r.cropp@griffith.edu.au (R. Cropp). 0304-3800/\$ - see front matter ( 2006 Elsevier B.V. All rights reserved. doi:10.1016/j.ecolmodel.2006.09.021
} 
inherent dynamics of the GMSK model is important in understanding more detailed and realistic models which include spatial and depth variation and seasonal (external) forcings. This work also demonstrates that the sulphur compartments of the GMSK model may be considered as a separate submodel that is slaved to the nitrogen-based ecosystem model, and therefore when considering the determinants of DMS we need only consider the dynamics of the ecosystem model. The work of Cropp et al. (2004) also demonstrates that this ecosystem model can be further decomposed into two submodels: a nutrient-phytoplankton-zooplankton (NPZ) model and a bacteria-zooflagellate-nutrient (BFN) sub-model.

This GMSK non-linear ODE ecosystem model (which has four degrees of freedom) possesses a very stable and somewhat complicated and interesting limit cycle; that is, a periodic set of solutions that correspond to regular plankton blooms (Cropp et al., 2004). It is often thought that systems with three or more degrees of freedom should have chaotic solutions. We find that the biological parameters have to be changed significantly in order to find pseudo-chaotic behaviour, and that for the most part the model is robust in its behaviour. A key advantage of understanding our stable limit cycle is that it allows for the identification of the various complicated interactions and processes in the time-dependent non-linear dynamics. In particular, we explore the influence of mortality parameters that extend the time spent on one part of the limit cycle, or that shut off the phytoplankton bloom, or that limit the importance of the larger species in the system.

\subsection{Mortality parameters}

Examination of the influence of mortality parameters on ecosystem model dynamics is also interesting and important in the context of modelling ecosystem responses to climate change. It is reasonable to assume that most anthropogenically unperturbed ecosystems, and most organisms within them, are well adapted to their ambient environment, and in particular to the ambient temperature. Generally therefore, species are maintained near the centre of their ecological niches. Any change in climate (warming or cooling) will have the effect of moving species from the optimal position in their ecological niche; where they have a relatively high reproduction rate and a relatively low mortality rate, to a less-optimal position where, for instance, reproduction is reduced and mortality increased. The response of ecosystem models to increases in mortality rate is therefore useful as an indicator of the influence of climate change, and also of the suitability of particular ecosystem models for applications in Earth System Models used to predict global climate change.

Mortality terms have also been identified as being important determinants of ecosystem model dynamics (Steele and Henderson, 1992; Fulton et al., 2003). Although many forms of mortality have been proposed for use in ecosystem models, linear mortality forms are often used because they are simple, require only one parameter each (parameters are often poorly known), and there are little empirical data to support alternative forms (Edwards and Brindley, 1999). The highest trophic level mortality terms in ecosystem models are often referred to as 'closure terms' as they 'close' the nutrient cycle. The coefficients of closure terms have served as effective bifurcation parameters in studies of simple ecosystem dynamics (Edwards and Brindley, 1996, 1999; Edwards and Bees, 2001). The choice of the form of closure terms has also been shown to have an important influence on the dynamics of simple ecosystem models (Steele and Henderson, 1992; Edwards and Yool, 2000). These studies found that simple (i.e. three-compartment, often NPZ) ecosystem models with linear closure terms were more likely to exhibit oscillatory behaviour (i.e. have limit cycles) than were the same models with other closure terms. We therefore chose to examine the influence of all the linear mortality terms in the model.

The GMSK model presents an interesting case for the examination of the influence of linear closure terms as the GMSK limit cycle may be considered the sum of the dynamics of its sub-models even though the full problem is highly non-linear. It is also particularly useful for the examination of the influence of mortality terms on model dynamics as its component sub-models both have linear closure terms but have very different dynamics. The NPZ sub-model has an asymptotically stable steady state while the BFN sub-model has a limit cycle (Cropp et al., 2004).

We initially explore varying three mortality parameters of the GMSK model; $k_{10}$ which controls the influence of the bacteria mortality term $k_{10} B ; k_{13}$ which controls the influence of the zooflagellate mortality term $k_{123} \mathrm{~F}$ and is the linear closure term of the BFN sub-model; and $k_{19}$ which controls the influence of the zooplankton mortality term $k_{19} Z$ and is the linear closure term for the NPZ sub-model. We note that the influence of $k_{19}$ is quite different to that of the other linear mortality terms; unlike $k_{10}$ and $k_{13}$ which control the rate of switching between the sub-models $k_{19}$ has no effect on the switching behaviour.

We examine the influence of varying these parameters over a substantial range (4-1000\% of measured values). Species in their optimal position have a low mortality rate compared to their reproductive rate: moving them from this position has the effect of lowering their reproduction rate and increasing their mortality rate. It is reasonable therefore, in a model in which the mortality rates are scaled relative to a reproduction rate, to examine the influence of quite large increases in mortality rates. The robustness of the model dynamics, in which the limit cycle or attractor is maintained over large regions of the parameter space, and only subtly changes shape in response to parameter variations, is of ecological interest.

\subsection{GMSK and climate models}

The production of DMS in the GMSK model is determined by the temporal dynamics of the ecosystem model as it circulates on or approaches its limit cycle, that is the relative time spent on different parts of the limit cycle determines how much DMS is produced. Production of DMS mostly occurs when the model is in the part of the limit cycle associated with the NPZ submodel, while DMS is mostly consumed when the model is in the part of the limit cycle associated with the BFN sub-model. Knowledge of the temporal dynamics of the attractor is therefore important for applications of the model in climate change studies.

The results of this research have several ecological implications. With respect to the GMSK model's primary function 
of modelling DMS production, we find that the system spends approximately $95 \%$ of its time in DMS production mode. However we also find that the GMSK model's response to climate change scenarios is very sensitive to the representation of mortality terms. The GMSK model and a subtly amended GMSK model make quite different predictions about ecosystem responses to climate change: the former predicts an autotroph-dominated ecosystem, while the latter predicts the extinction of all life. The primary function of our GMSK model is to estimate DMS production by ecological systems when subject to climate change. This is because DMS production itself feeds back on climate change. The above-mentioned two outcomes predict profoundly different DMS production in warmer climates: the first autotroph-dominated outcome predicts a continuous, strong negative feedback on global warming, whereas the second outcome predicts the extinction of life and a run-away greenhouse effect.

This result suggests that great care must be taken in selecting ecological models used to predict the impacts of events such as climate change that affect the processes represented in the models. For more local systems, our results suggest that the addition of nutrients to otherwise stable plankton ecosystems (as humans invariably do with sewage discharges and agricultural run-off) could fundamentally change the dynamics of those ecosystems from phytoplankton-controlled to microbe-controlled.

Of independent mathematical interest, we see that the flow of this four-dimensional system always lies on the intersection of the positive orthant in $B, F, N, P, Z$ space with the hyperplane $B+F+N+P+Z=1$ (the state variables are normalised so that the total amount of matter in nitrogen currency is 1$)$. This four-dimensional system together with possible parameter variation and solution state bifurcation, for most parameter values, appears to possess a very stable periodic orbit with dramatically different behaviour on different parts of the (highly non-linear) orbit. Asymptotically stable states and apparently chaotic motion can also be found in the parameter regimes that we explored. We finally note that the attributes of the GMSK model are largely described by the sum of the attributes of its sub-models in spite of the non-linear linkages of these models and their (highly) non-linear solutions.

\section{The GMSK model}

The ecological part of the GMSK model (Gabric et al., 1993) is composed of five biotic compartments: phytoplankton $(P)$, zooplankton $(Z)$, dissolved inorganic nitrogen $(N)$, bacteria $(B)$ and zooflagellates $(F)$. The five coupled differential equations of our GMSK model, together with the mass conservation constraint $P+Z+N+B+F=1$, form a four degrees of freedom system where each variable is a concentration satisfying $0 \leq P$, $Z, N, B, F \leq 1$ for all time $t \geq 0$. We focus on a conservative system to analyse the nutrient transformation behaviour (the losses and gains of nutrient in the system are looked at in the spatially resolved model, see for instance Cropp et al., 2004), since significant understanding arises from considering the self-contained, non-externally forced GMSK model see Bazykin (1998) for further discussion of this view.
The GMSK model is composed of the following equations:

$$
\frac{\mathrm{dP}}{\mathrm{dt}}=k_{23}\left(\frac{N}{N+k_{24}}\right) P-k_{1}\left(\frac{P}{P+k_{2}}\right) B-k_{4} P Z,
$$

$$
\begin{aligned}
\frac{d B}{d t}= & k_{1}\left(1-k_{11}\right)\left(\frac{P}{P+k_{2}}\right) B+k_{25}\left(1-k_{11}\right)\left(\frac{N}{N+k_{26}}\right) B \\
& -k_{8}\left(\frac{B}{B+k_{9}}\right) F-k_{10} B,
\end{aligned}
$$

$$
\frac{\mathrm{dF}}{\mathrm{dt}}=k_{8}\left(1-k_{14}\right)\left(\frac{B}{B+k_{9}}\right) F-k_{13} F,
$$

$$
\frac{d Z}{d t}=k_{4}\left(1-k_{20}\right) P Z-k_{19} Z
$$

$$
\begin{aligned}
\frac{\mathrm{dN}}{\mathrm{dt}}= & k_{10} B+k_{11}\left[k_{25}\left(\frac{N}{N+k_{26}}\right) B+k_{1}\left(\frac{P}{P+k_{2}}\right) B\right]+k_{13} F \\
& +k_{8} k_{14}\left(\frac{B}{B+k_{9}}\right) F+k_{19} Z+k_{4} k_{20} P Z-k_{23}\left(\frac{N}{N+k_{24}}\right) P \\
& -k_{25}\left(\frac{N}{N+k_{26}}\right) B .
\end{aligned}
$$

These equations for the rates of change of the biotic components have non-linear source/sink terms that are either the product of active agents (Lotka-Volterra predation terms) or have Michaelis-Menten (Holling type II) substrate-dependent growth modifiers that are conventional in ecological modelling.

The parameter set defining the most "realistic" instantiation of the GMSK ecosystem model (Table 1) is composed of nine contemporaneously measured parameter values from station one of Gabric et al. (1999) with another six values measured at varying times and places taken from the literature values cited by Gabric et al. (1993).

Eqs. (1)-(5) are non-dimensionalised and the state variables replaced by their scaled equivalents: $C^{\prime}=C / N_{0}$ for $C=B, F, N$, $P$ and $Z$ while time is scaled by the maximum phytoplankton growth rate $t^{\prime}=k_{23} t$. The parameters are then replaced by their dimensionless equivalents as defined by Eqs. (6)-(9). The resultant scaled parameter values are listed in the final column of Table 1. These values will be used throughout this analysis unless otherwise specified.

$k_{i}^{\prime}=\frac{k_{i}}{k_{23}}, \quad$ for $i=1,8,10,13,19,23$ and 25,

$k_{j}^{\prime}=\frac{k_{j}}{N_{0}}, \quad$ for $j=2,9,24$, and 26,

$k_{l}^{\prime}=\frac{k_{l} N_{0}}{k_{23}}, \quad$ for $l=4$,

$k_{n}^{\prime}=k_{n}, \quad$ for $n=11,14$ and 20

The non-dimensionalization implemented on the GMSK model scales the mortality rates of $B, F$ and $Z$ relative to the maximum $P$ growth rate. When investigating the effects of 
Table 1 - Measured ecosystem parameter values for the GMSK model

\begin{tabular}{|c|c|c|c|}
\hline PAR & Process & Measured value & Scaled value \\
\hline$k_{1}$ & Maximum rate of $N$ uptake by $B\left(\right.$ day $\left.^{-1}\right)$ & 0.31 & 1.148 \\
\hline$k_{2}$ & Half-saturation constant for $\mathrm{B}$ uptake of $\mathrm{N}\left(\mathrm{mg} \mathrm{Nm}^{-3}\right)$ & 34.65 & 0.693 \\
\hline$k_{4}$ & $Z$ grazing rate (per individual) on $P\left(\mathrm{~m}^{3} \mathrm{mg} \mathrm{N}^{-1}\right.$ day $\left.^{-1}\right)$ & 0.01 & 1.852 \\
\hline$k_{8}$ & Maximum rate of $B$ uptake by $F\left(\right.$ day $\left.^{-1}\right)$ & 1.67 & 6.185 \\
\hline$k_{9}$ & Half-saturation constant for $F$ uptake of $B\left(\mathrm{mg} \mathrm{Nm}^{-3}\right)$ & 9.10 & 0.182 \\
\hline$k_{10}$ & B mortality $\left(\right.$ day $\left.^{-1}\right)$ & 0.07 & 0.259 \\
\hline$k_{11}$ & Proportion of $N$ uptake excreted by $B$ & 0.63 & 0.630 \\
\hline$k_{13}$ & F mortality $\left(\right.$ day $\left.^{-1}\right)$ & 0.05 & 0.185 \\
\hline$k_{14}$ & Proportion of $\mathrm{N}$ uptake excreted by $\mathrm{F}$ & 0.65 & 0.650 \\
\hline$k_{19}$ & Z mortality $\left(\right.$ day $\left.^{-1}\right)$ & 0.05 & 0.185 \\
\hline$k_{20}$ & Proportion of $\mathrm{N}$ uptake excreted by $\mathrm{Z}$ & 0.40 & 0.400 \\
\hline$k_{23}$ & Maximum rate of $N$ uptake by $P\left(\right.$ day $\left.^{-1}\right)$ & 0.27 & 1.000 \\
\hline$k_{24}$ & Half-saturation constant for $\mathrm{P}$ uptake of $\mathrm{N}\left(\mathrm{mg} \mathrm{Nm}^{-3}\right)$ & 12.60 & 0.252 \\
\hline$k_{25}$ & Maximum rate of $N$ uptake by $B\left(\right.$ day $\left.^{-1}\right)$ & 0.31 & 1.148 \\
\hline$k_{26}$ & Half-saturation constant for B uptake of $\mathrm{N}\left(\mathrm{mg} \mathrm{Nm}^{-3}\right)$ & 3.45 & 0.069 \\
\hline$N_{0}$ & Total nutrient $(\mathrm{N})$ as nitrogen $\left(\mathrm{mg} \mathrm{Nm}^{-3}\right)$ & 154 & 1 \\
\hline
\end{tabular}

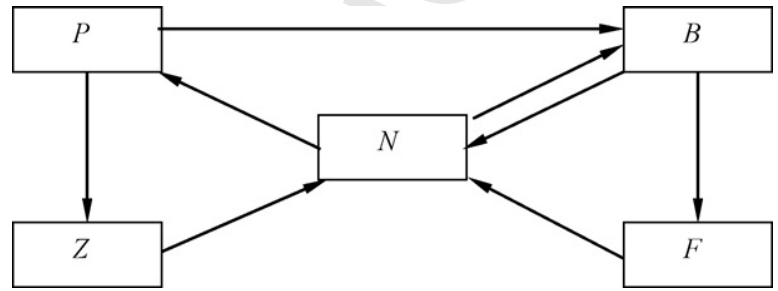

Fig. 1 - Schematic of the GMSK model. Arrows represent the direction of mass flows as $B$ predates on $P$ and so on. between Mode A and Mode B of the GMSK model may therefore have significant consequences for the climate predictions that the model produces.

\section{Analysis}

\subsection{Dynamics}

The non-transient dynamics of the GMSK model are confined to a very structurally stable and highly non-linear periodic orbit shown in NPZ and BFN state spaces in Figs. 2 and 3. The planes defined by the conservation of mass criterion in each state space $\left(N+P+Z=1\right.$ and $B+F+N=1$ together with $0 \leq C_{i} \leq 1$ for $C_{i}=B, F, N, P$ and $Z$ ) are shown by dotted black lines marking out triangles in Figs. 2 and 3, respectively. The stars in these figures indicate the location of the model's critical points. The arrows show the direction of the eigenvectors of the largest 


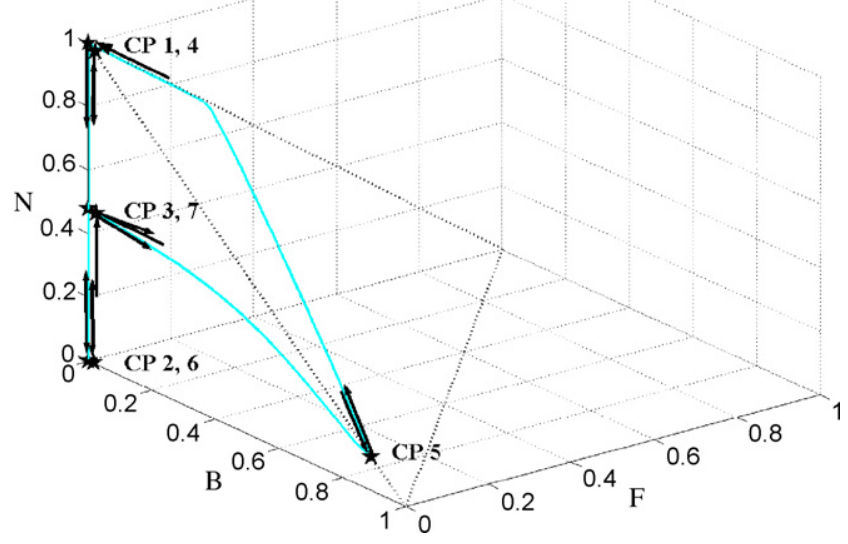

Fig. 3 - Periodic orbit of the GMSK model shown in BFN state space. Trajectories and eigenvectors as for Fig. 2.

\section{Table 2 - Locations of critical points of the GMSK model} for measured parameter values

\begin{tabular}{llllll}
$\mathrm{CP}$ & \multicolumn{1}{c}{$\mathrm{B}^{*}$} & \multicolumn{1}{c}{$\mathrm{F}^{*}$} & \multicolumn{1}{c}{$\mathrm{N}^{*}$} & \multicolumn{1}{c}{$\mathrm{P}^{*}$} & \multicolumn{1}{c}{$Z^{*}$} \\
\hline 1 & 0 & 0 & 1.000 & 0 & 0 \\
2 & 0 & 0 & 0 & 1.000 & 0 \\
3 & 0 & 0 & 0.4794 & 0.1667 & 0.3539 \\
4 & 0.0170 & 0.0044 & 0.9785 & 0 & 0 \\
5 & 0.8919 & 0 & 0.1081 & 0 & 0 \\
6 & 0.0170 & 0.0002 & 0.0030 & 0.9798 & 0 \\
7 & 0.0170 & 0.0062 & 0.4707 & 0.1667 & 0.3394 \\
\hline
\end{tabular}

magnitude positive and negative eigenvalues at each point for the measured parameter values shown in Table 1 . The values of the critical points, eigenvalues and eigenvectors are shown in Tables 2-4, respectively. The importance of these attributes will be discussed later.

Figs. 2 and 3 clearly demonstrate the existence of the two components of the GMSK limit cycle, a spiral in NPZ space, that we have named Mode A, and a limit cycle in BFN space that we have named Mode B. The model structure shown in Fig. 1 also reveals that the GMSK model may be separated into two sub-models, a BFN sub-model and an NPZ sub-model, by the deletion of the link representing $B$ predation on $P$. These observations suggest that it may be useful to first examine these sub-models to explain the behaviour observed in the complete GMSK model.

We find that the ability of the bacteria to act on the phytoplankton bloom is crucial to understanding the overall behaviour of the system, and that the limit cycle is essentially the result of coupling an autotrophic and a heterotrophic system. It also eventuates that the $B$ and $F$ mortality terms control the switching between Mode A and Mode B.

\subsection{Switching behaviour}

Figs. 2 and 3 do not reveal an important feature of the model, the fact that the system spends very different amounts of time in the respective modes. This is evident in time series such as Fig. $4 \mathrm{a}$ and $\mathrm{b}$, where the system is in Mode A when oscillations in the panel (a) are decaying towards an equilibrium, and in Mode $B$ when the $B$ and $F$ populations undergo bloom events (see panel (b)) that comprise a single bloom-and-bust cycle that "resets" the oscillatory decay of the $P$ and $Z$ blooms in Mode A.

The system flips from Mode A to Mode B when B escapes the control of $F$. As the maximum rate of $F$ predation on $B\left(k_{8}\right)$ is relatively large, and near maximum growth is achieved at low $B$ levels due to the relatively low value of $F^{\prime} s$ half-saturation constant of $B\left(k_{9}\right)$, this only occurs after both $B$ and $F$ have fallen to very low levels and the $F$ growth term $k_{8}\left(B / B+k_{9}\right) F$ in Eq. (2) is very small. As $F$ will graze $B$ to low levels rapidly due to the relatively large value of $k_{8}$ this time scale will essentially be controlled by the $F$ mortality term $k_{13}$, as at low levels of $B$, F will decay like:

$F=F_{B_{\min }} e^{-k_{13}\left(t-t_{B_{\min }}\right)}$

Once $F$ has decayed to near-zero $B$ can begin to grow and switch the system from Mode A to Mode B. The timing of this switching is therefore dependent on the magnitude of $k_{13}$ (Fig. $4 \mathrm{e}$ and f). During the period that $B$ is prevented from blooming by $F$, the system has approached the centre of the spiral in Fig. 2. At this point $N, P$ and $Z$ are approximately constant and $F$ is approximately zero. From Eq. (2) we can see that $B$ growth can now be described by:

$\frac{\mathrm{dB}}{\mathrm{dt}}=\left(C^{*}-k_{10}\right) \mathrm{B}$,

where $C^{*}$ is a constant and represents the coefficients of the first two terms in Eq. (2), and the term involving $F$ is effectively zero. The initiation of the $B$ bloom that flips the system from Mode A to Mode B then evolves as:

$B=B^{*} e^{\left(C^{*}-k_{10}\right)\left(t-t^{*}\right)}$

Table 3 - Eigenvalues of critical points for measured parameter values

\begin{tabular}{|c|c|c|c|c|}
\hline $\mathrm{CP}$ & \multicolumn{4}{|c|}{ Eigenvalues } \\
\hline 1 & -0.1852 & 0.7987 & 0.1381 & -0.1852 \\
\hline 2 & -0.1852 & -3.9683 & -0.0083 & 0.9259 \\
\hline 3 & $-0.0393+0.3882 i$ & $-0.0393-0.3882 i$ & -0.1852 & 0.1945 \\
\hline 4 & $0.0057+0.1526 i$ & $0.0057-0.1526 i$ & 0.7670 & -0.1852 \\
\hline 5 & -0.8340 & 1.6128 & -1.1777 & -0.1852 \\
\hline 6 & -3.9017 & $0.0076+0.0335 i$ & $0.0076-0.0335 i$ & 0.9034 \\
\hline 7 & $-0.0384+0.3839 i$ & $-0.0384-0.3839 i$ & $0.0079+0.1798 i$ & $0.0079-0.1798 i$ \\
\hline
\end{tabular}


Table 4 - Eigenvectors of critical points for measured parameter values

\begin{tabular}{|c|c|c|c|c|}
\hline $\mathrm{CP}$ & & & & \\
\hline & 0 & 0 & 1 & 0 \\
\hline & 0 & 0 & 0 & 1 \\
\hline 1 & 0 & 1 & 0 & 0 \\
\hline & 1 & 0 & 0 & 0 \\
\hline & 0 & 0 & 0.6446 & 0 \\
\hline ? & 0.6896 & 0 & 0 & 0.6389 \\
\hline 2 & -0.7234 & 1 & -0.7563 & -0.7598 \\
\hline & 0 & 0 & 0 & 0 \\
\hline & 0 & 0 & 0 & 0.8354 \\
\hline 2 & 0 & 0 & 0.7335 & 0 \\
\hline 3 & $0.3411+0.3806 i$ & $0.3411-0.3806 i$ & 0.0620 & -0.2382 \\
\hline & $0.3471-0.3806 i$ & $0.3471+0.3806 i$ & -0.1317 & -0.4817 \\
\hline & $-0.6502-0.1854 i$ & $-0.6502+0.1854 i$ & 0.0089 & 0.0010 \\
\hline & $-0.0604+0.1854 i$ & $-0.0604-0.1854 i$ & 0.0005 & -0.0002 \\
\hline 4 & 0 & 0 & 0.7023 & 0 \\
\hline & 0 & 0 & 0 & 0.7067 \\
\hline & 1 & -0.8121 & 0.3713 & -0.7774 \\
\hline 5 & 0 & 0.3328 & 0 & 0 \\
\hline o & 0 & 0 & 0.4441 & 0.6048 \\
\hline & 0 & 0 & 0 & 0.6048 \\
\hline & 0.0173 & $-0.6369-0.0403 i$ & $-0.6369+0.0403 i$ & 0.0116 \\
\hline 6 & 0 & $-0.0120+0.0407 i$ & $-0.0120-0.0407 i$ & 0 \\
\hline 0 & 0.6983 & 0.7609 & 0.7609 & -0.7657 \\
\hline & 0 & 0 & 0 & 0.6312 \\
\hline & $-0.0075+0.0055 i$ & $-0.0075-0.0055 i$ & -0.6136 & -0.6136 \\
\hline 7 & $0.0010+0.0011 i$ & $0.0010-0.0011 i$ & $-0.0092+0.2110 i$ & $-0.0092-0.2110 i$ \\
\hline$\gamma$ & $0.3476-0.3819 i$ & $0.3476+0.3819 i$ & $-0.0793+0.2793 i$ & $-0.0793-0.2793 i$ \\
\hline & $-0.3376+0.3753 i$ & $-0.3376-0.3753 i$ & $0.5918-0.1405 i$ & $0.5918+0.1405 i$ \\
\hline
\end{tabular}

Variations in the parameter $k_{10}$ can therefore also have a significant effect on the timing of the switching between Mode A and Mode B of the GMSK model (Fig. 4c and d). Increases in $k_{10}$ (i.e. $k_{10} \rightarrow C^{*}$ ) cause the system to spend a longer period in Mode A before flipping into Mode B. There are therefore at least two characteristic time scales operating in the model to flip the system dynamics between Mode A and Mode B. After the system has attracted to the vicinity of $\mathrm{CP} 3$ (see Fig. 2), and $N, P$ and $Z$ are relatively unchanging, the transition from Mode A to Mode B, related to the decay of the $F$ population and subsequent bloom of the $B$ population, occurs on a time scale proportional to $k_{10}$ and inversely proportional to $k_{13}$.

Variations in $\mathrm{Z}$ mortality cause the model to respond in a very different manner to variations in the other two mortality rates (Fig. 5). Although $k_{19}$ does not affect the switching behaviour, it can affect the timing of the system dynamics, with the frequency of oscillation increasing as $k_{19}$ is reduced. This parameter also has other, rather surprising effects; an interesting consequence of reducing $k_{19}$ is that the system appears to gain a "memory". Fig. $4 a$ and $b$ shows that for measured parameter values the system "resets" to a state near CP 1 (see Fig. 2) where the biotic compartments are very small, and nearly everything exists as inorganic nutrient. However, Fig. $5 a$ and $b$ reveals that when $k_{19}$ is very small, after the BFN cycle has perturbed the NPZ cycle, the system returns to a point on its NPZ trajectory where it would have been had it not been perturbed.

The system now exhibits a new Mode $C$ that might be described as a combination of Modes A and B. Mode $C$ is essentially a BFNZ system: after a brief $P$ bloom $Z$ consumes $P$ and then decays like $e^{-k_{19} t}$ while $N$ grows like $e^{k_{19} t}$. This transfer is intermittently perturbed by $B$ growing on $N$, which subsequently initiates an $F$ bloom that rapidly consumes all of the $B$ resource before decaying as $\mathrm{e}^{-k_{13} t}$. After $F$ has decayed the system returns to near its previous position in the $\mathrm{N}-\mathrm{Z}$ transfer, in fact the place it would have been had it not been perturbed by the $B$ bloom. Mode $C$ therefore exhibits a "memory" where it returns from Mode $B$ to its previous position rather than resetting via CP 1.

Reduction of $k_{19}$ also pushes the system into a state that appears potentially chaotic (Fig. $5 \mathrm{c}$ and d). However, numerical calculation of the Lyapunov exponents of the system for low values of $k_{19}$ using the method of Wolfe et al. (1985) reveals that the system at no time becomes truly chaotic. Fig. 5e and $\mathrm{f}$ also reveals that as $k_{19}$ is increased the resilience, a measure of the rate at which the system attracts back to the critical point after a perturbation (DeAngelis, 1980), of the NPZ system of Mode A increases dramatically. This further emphasises the importance of $k_{19}$ on the time scale of the $\mathrm{Z}$ to $\mathrm{N}$ nutrient transfer. 

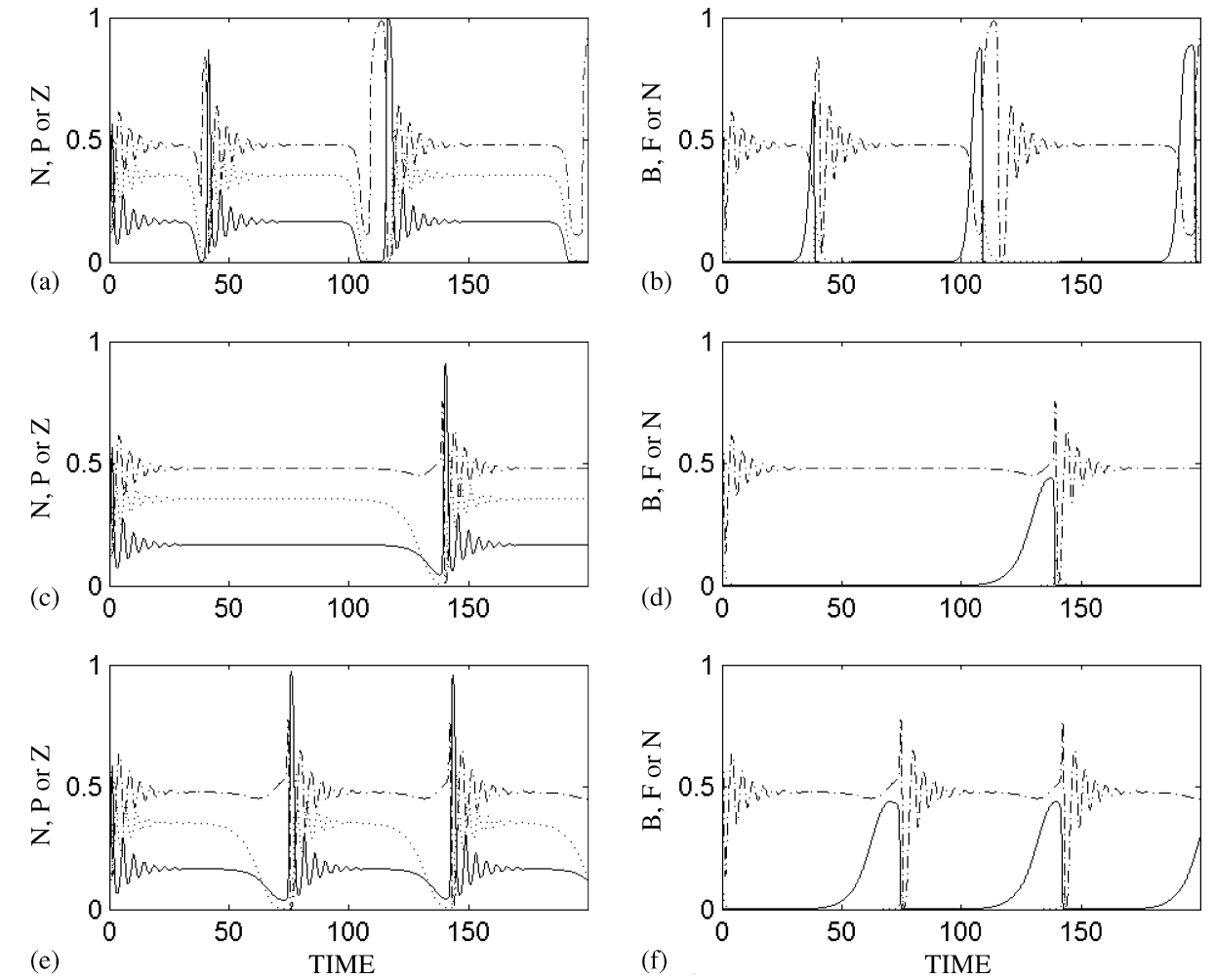

Fig. 4 - The time series of the GMSK model for measured parameter values (a and b); with $k_{10}$ increased to 0.40 (c and $d$ ); and with $k_{13}$ increased to 0.40 (e and f). Panels (a, c and e) show P (solid line), Z (dotted line) and N (dashed line). Panels (b, d and f) show $B$ (solid line), $F$ (dotted line) and $N$ (dashed line). The system was integrated for 200 time periods from the initial condition $[B, F, N, P, Z]=[0.1,0.1,0.6,0.1,0.1]$.
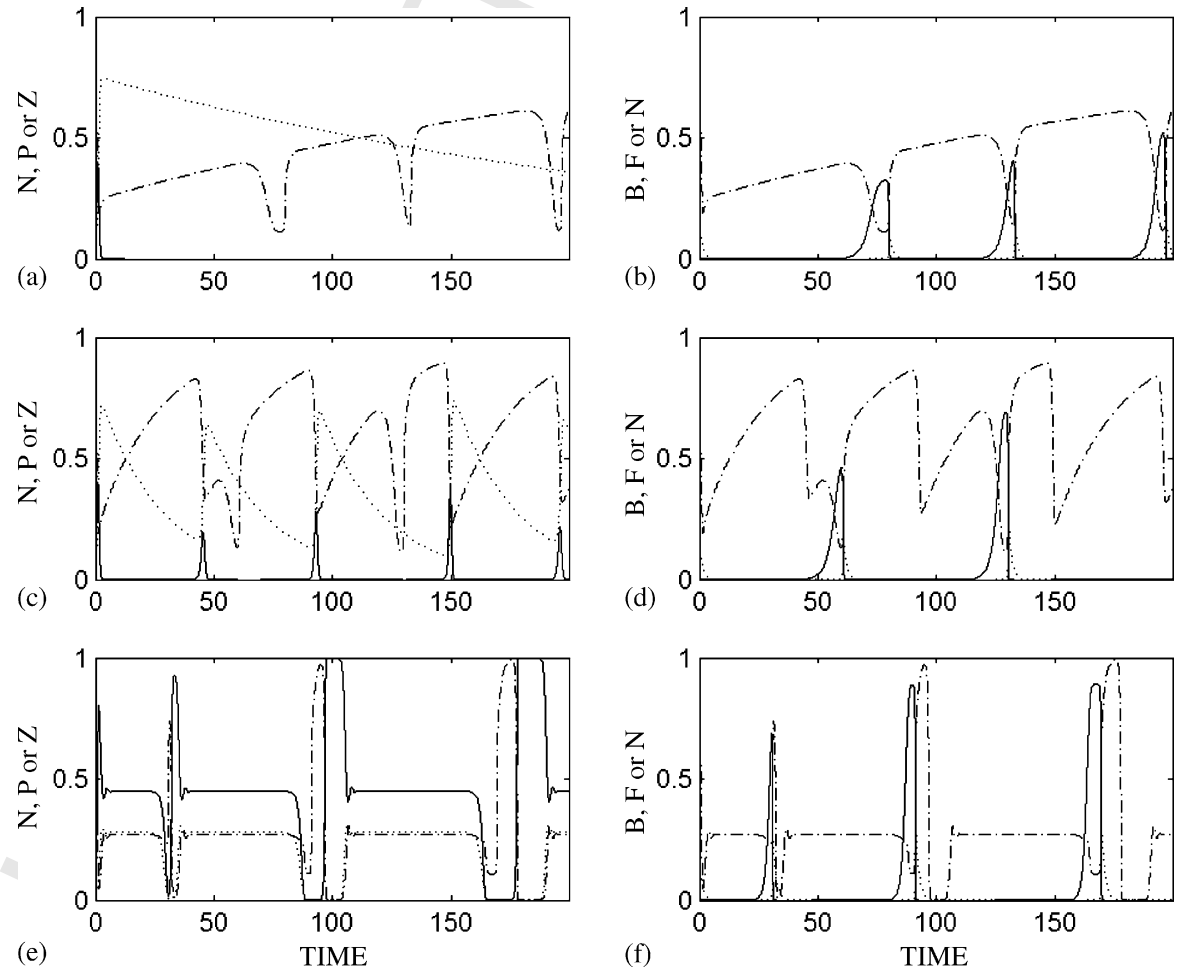

Fig. 5 - Time series behaviour of the GMSK model for: (a and b) $k_{19}=0.001$, (c and d) $k_{19}=0.01$ and $(e$ and $f) k_{19}=0.50$. Integrations and panel layout are as for Fig. 4. 


\subsection{NPZ sub-model}

The GMSK model may be broken into two sub-models by severing the link between $P$ and $B$ and allowing each submodel unfettered access to nutrient, $N$. Each of the sub-models exhibits dynamics typical of either Mode A or Mode B. The NPZ sub-model, which encapsulates the dynamics typical of Mode A, comprises the system:

$\frac{d P}{d t}=k_{23}\left(\frac{N}{N+k_{24}}\right) P-k_{4} P Z$,

$\frac{d Z}{d t}=k_{4}\left(1-k_{20}\right) P Z-k_{19} Z$,

$\frac{\mathrm{dN}}{\mathrm{dt}}=k_{19} Z+k_{4} k_{20} \mathrm{PZ}-k_{23}\left(\frac{\mathrm{N}}{\mathrm{N}+k_{24}}\right) P$.

The eigenvalues and associated eigenvectors of the critical points of the NPZ sub-model may be obtained analytically from its Jacobian matrix (Appendix A). For measured parameter values the sub-model has a critical point (CP 1) at the origin of the system where no biota are extant:

$N_{1}^{*}=1$

$P_{1}^{*}, Z_{1}^{*}=0$

The eigenvalues of the linearised NPZ sub-model about CP 1 are:

$\lambda_{N P Z \_1-1}=k_{23}\left(\frac{N_{1}^{*}}{N_{1}^{*}+k_{24}}\right)$,

$\lambda_{\text {NPZ_1-2 }}=-k_{19}$.

As $\lambda_{\text {NPZ_1-1 }}$ is always positive and $\lambda_{\text {NPZ_1-2 }}$ is always negative, CP 1 is always a saddle point in the NPZ sub-model. A second critical point (CP 2) is the autotroph-only critical point:

$P_{2}^{*}=1$

$Z_{2}^{*}, N_{2}^{*}=0$

The eigenvalues of the linearised NPZ sub-model around CP 2 are:

$\lambda_{\mathrm{NPZ} \_-1}=-\frac{k_{23}}{k_{24}}$

$\lambda_{\mathrm{NPZ} \_2-2}=k_{4}\left(1-k_{20}\right)-k_{19}$

$\lambda_{\text {NPZ_2-1 }}$ is always negative and $\lambda_{\text {NPZ_2-2 }}$ is positive for measured parameter values but negative for:

$k_{19}>k_{4}\left(1-k_{20}\right)=1.111$

CP 2 is therefore a saddle point for measured values but will be a stable node in the NPZ sub-model when $k_{19}>1.111$.
$P_{3}^{*}=\frac{k_{19}}{k_{4}\left(1-k_{20}\right)}$,

$Z_{3}^{*}=\frac{k_{23}}{k_{4}}\left(\frac{N_{3}^{*}}{N_{3}^{*}+k_{24}}\right)$,

$N_{3}^{*}=\frac{1}{2}\left[-\left(k_{24}+P_{3}^{*}+\frac{k_{23}}{k_{4}}-1\right)\right.$

$\left.\pm \sqrt{\left(k_{24}+P_{3}^{*}+\frac{k_{23}}{k_{4}}-1\right)^{2}-4 k_{24}\left(P_{3}^{*}-1\right)}\right]$,

where only the positive root of Eq. (27) is considered. The eigenvalues of the linearised NPZ sub-model about CP 3 are:

$$
\begin{aligned}
& \lambda_{\mathrm{NPZ}_{-}-1,2}=-\frac{k_{23} \mathrm{P}_{3}^{*}}{2}\left[\frac{k_{24}}{\left(N_{3}^{*}+k_{24}\right)^{2}}\right] \\
& \times\left\{1 \pm \sqrt{1-\left(N_{3}^{*}+k_{24}\right)\left[1+\frac{\left(N_{3}^{*}+k_{24}\right)^{2}}{k_{23} k_{24} \mathrm{P}_{3}^{*}}\right]\left(\frac{4 k_{19} N_{3}^{*}}{k_{24} \mathrm{P}_{3}^{*}}\right)}\right\} .
\end{aligned}
$$

The stability of this critical point will depend on the value of the discriminant ( $\left.\chi_{\mathrm{NPZ} 3-1,2}\right)$ of the eigenvalues:

$\chi_{\text {NPZ } 3-1,2}=\left(N_{3}^{*}+k_{24}\right)\left[1+\frac{\left(N_{3}^{*}+k_{24}\right)^{2}}{k_{23} k_{24} P_{3}^{*}}\right]\left(\frac{4 k_{19} N_{3}^{*}}{k_{24} P_{3}^{*}}\right)$

CP 3 would be a saddle point if $\chi_{\text {NPZ_3-1,2 }}<0$, but Eq. (29) reveals this is not possible in the ecologically feasible region of state space. The dynamics of the steady state for $\chi_{\text {NPZ_3-1,2 }}>0$ are:

- proper node, if $0<\chi_{\text {NPZ } 3-1,2}<1$,

- node, if $\chi_{\text {NPZ_-3 }-1.2}=1$,

- spiral point, if $\chi_{\mathrm{NPZ}-3-1,2}>1$ (Boyce and DiPrima, 1997).

The dynamics near CP 3 will be stable for all $\chi_{\mathrm{NPZ}-3-1,2}>0$, as the real part of the eigenvalues, given by:

$\operatorname{Re}\left(\lambda_{\mathrm{NPZ}_{3}-1,2}\right)=-\frac{k_{23}}{2}\left[\frac{k_{24}}{\left(N_{3}^{*}+k_{24}\right)^{2}}\right] P_{3}^{*}$,

is always negative. Therefore CP 3 is always stable in the NPZ sub-model and is a spiral point for measured parameter values, but may be a node.

The NPZ model does not include $k_{10}$ or $k_{13}$, and is sensitive only to variations in the value of $k_{19}$. Changes in $k_{19}$ affect the location of only one of the three critical points (CP 3) but the eigenvalues of all the critical points. While variations in $k_{19}$ affect $\lambda_{\text {NPZ_1-1,2 }}$, they do not affect the signs of the eigenvalues and CP 1 always remains a saddle. However, increasing $k_{19}$ above the measured value leads to a transcritical bifurcation where CP 3 collides with CP 2 at $P_{2}^{*}=1$ and they exchange stability. It is apparent from Eqs. (23) and (25) that this occurs when:

$k_{19}=k_{4}\left(1-k_{20}\right)=1.111$
452 


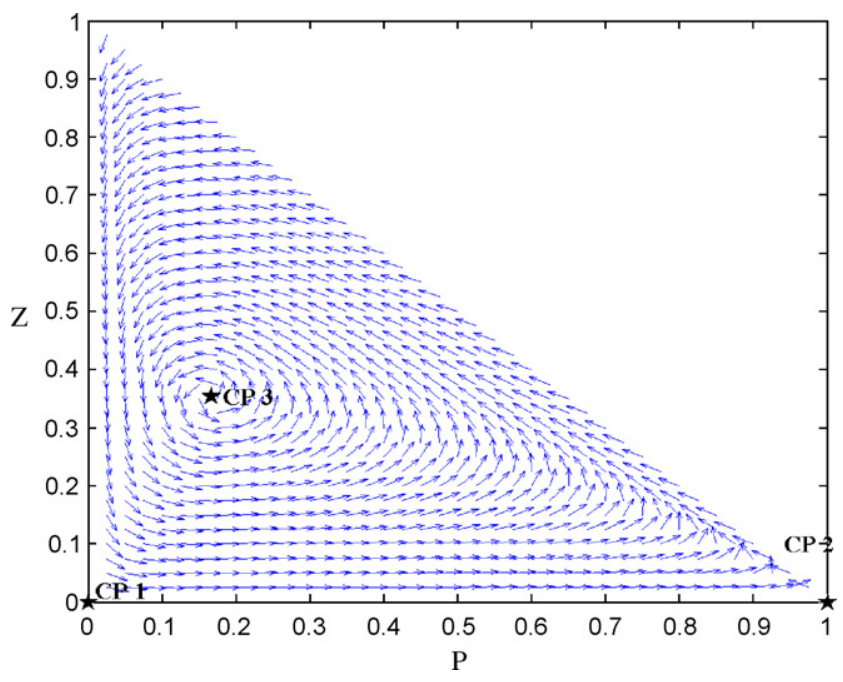

Fig. 6 - The direction field of the NPZ model shown in PZ state space. As $k_{19}$ increases CP 3 moves toward CP 2 and reaches it when $k_{19}=k_{4}\left(1-k_{20}\right)=1.111$.

At this point the discriminant of the eigenvalues of CP 3 (Eq. (29)) has reduced to zero, and as $k_{19}$ is increased further CP 3 leaves the feasible region and becomes a saddle point as $\chi_{\text {NPZ } 3-1,2}$ becomes negative. The value of CP 2 is unaffected by further increases in $k_{19}$ and remains a feasible, stable node.

The vector field for the NPZ sub-model for measured parameter values is shown in Fig. 6 . This reveals that the initial flow is away from the origin (CP 1) towards CP 2 where $P$ contains all the nutrient in the sub-model. The flow from CP 2 curves slightly inward from the mass closure boundary $P+Z=1$ and around $C P 3$. The attracting eigenvalues of this point specify a slowly decaying spiral node: we see in Table 4 that the real parts of the eigenvalues of CP 3 are an

Analytic expressions for the eigenvalues and associated eigenvectors of the critical points of the BFN sub-model may be obtained from its Jacobian matrix (Appendix B). For measured parameter values the BFN sub-model has a critical point at the origin of the system where no biota are extant that we will label CP 1 as for the NPZ sub-model:

$N_{1}^{*}=1$

$B_{1}^{*}, F_{1}^{*}=0$

The eigenvalues of the linearised BFN sub-model about CP 1 are:

$\lambda_{\text {BFN_1-1 }}=k_{25}\left(1-k_{11}\right)\left(\frac{N_{1}^{*}}{N_{1}^{*}+k_{26}}\right)-k_{10}$,

$\lambda_{\text {BFN_1-2 }}=-k_{13}$.

$\lambda_{B F N-1-1}$ is negative for:

$k_{10}>\frac{k_{25}\left(1-k_{11}\right) N_{1}^{*}}{N_{1}^{*}+k_{26}}=0.3973$,

and $\lambda_{B F N .1-2}$ is always negative, therefore CP 1 is a saddle point for measured parameter values but is a stable node for $k_{10}>0.3973$.

The second critical point of the BFN sub-model (CP 4) is located at:

$B_{4}^{*}=\frac{k_{9} k_{13}}{k_{8}\left(1-k_{14}\right)-k_{13}}$,

$F_{4}^{*}=\frac{k_{25}}{k_{8}}\left(1-k_{11}\right)\left(B_{4}^{*}+k_{9}\right)\left(\frac{N_{4}^{*}}{N_{4}^{*}+k_{26}}\right)-\frac{k_{10}}{k_{8}}\left(B_{4}^{*}+k_{9}\right)$,

$$
\pm \sqrt{\left(k_{26}+B_{4}^{*}+\left(\frac{B_{4}^{*}+k_{9}}{k_{8}}\right)\left(k_{25}\left(1-k_{11}\right)-k_{10}\right)-1\right)^{2}-4 k_{26}\left(B_{4}^{*}-1+k_{10}\left(\frac{B_{4}^{*}+k_{9}}{k_{8}}\right)\right)} \text {, }
$$

order of magnitude smaller than the imaginary parts. This means that orbits spiral around CP 3 many times, being attracted in towards CP 3 only very slowly.

\subsection{BFN sub-model}

The BFN sub-model, which exhibits the dynamics typical of Mode $B$, is given by:

$\frac{\mathrm{dB}}{\mathrm{dt}}=k_{25}\left(1-k_{11}\right)\left(\frac{N}{N+k_{26}}\right) B-k_{8}\left(\frac{B}{B+k_{9}}\right) F-k_{10} B$,

$\frac{d F}{d t}=k_{8}\left(1-k_{14}\right)\left(\frac{B}{B+k_{9}}\right) F-k_{13} F$,

$\frac{d N}{d t}=k_{10} B-k_{25}\left(1-k_{11}\right)\left(\frac{N}{N+k_{26}}\right) B+k_{13} F+k_{8} k_{14}\left(\frac{B}{B+k_{9}}\right) F$. where only the positive root of Eq. (42) is considered. The eigenvalues of the linearised BFN sub-model about CP 4 are:

$$
\begin{aligned}
\lambda_{\text {BFN_4-1,2 }}= & {\left[k_{8}\left(\frac{B_{4}^{*}}{\left(B_{4}^{*}+k_{9}\right)^{2}}\right)-k_{25}\left(1-k_{11}\right)\left(\frac{k_{26}}{\left(N_{4}^{*}+k_{26}\right)^{2}}\right) B_{4}^{*}\right] } \\
& \times\left\{1 \pm \sqrt{\left.1-\chi_{\text {BFN_4 }}\right\}}\right.
\end{aligned}
$$

where $\chi_{B N F} 4$ is the discriminant of the eigenvalues, and:

$$
\begin{aligned}
& 4\left[k_{25}\left(1-k_{11}\right)\left(k_{26} /\left(N_{4}^{*}+k_{26}\right)^{2}\right) B_{4}^{*}+k_{8}\left(B_{4}^{*} / B_{4}^{*}+k_{9}\right)\right] \\
& \chi_{\text {BFN_4 }}=\frac{\left[k_{8}\left(1-k_{14}\right)\left(k_{9} /\left(B_{4}^{*}+k_{9}\right)^{2}\right) F_{4}^{*}\right]}{\left[k_{8}\left(B_{4}^{*} /\left(B_{4}^{*}+k_{9}\right)^{2}\right) F_{4}^{*}-k_{25}\left(1-k_{11}\right)\left(k_{26} /\left(N_{4}^{*}+k_{26}\right)^{2}\right) B_{4}^{*}\right]^{2}} \text {. }
\end{aligned}
$$

The stability of this critical point of the BFN sub-model is subject to the same criteria as CP 3 of the NPZ sub-model described 
$F_{5}^{*}=0$

The eigenvalues of the linearised BFN sub-model about CP 5 are:

$\lambda_{\text {BFN_5-1 }}=k_{25}\left(1-k_{11}\right)\left(\frac{N_{5}^{* 2}+2 k_{26} N_{5}^{*}+k_{26}}{\left(N_{5}^{*}+k_{26}\right)^{2}}\right)-k_{10}$,

$\lambda_{\text {BFN }-5-2}=k_{8}\left(1-k_{14}\right)\left(\frac{B_{5}^{*}}{B_{5}^{*}+k_{9}}\right)-k_{13}$.

Evaluation of Eqs. (48) and (49) reveals that $\lambda_{\text {BNF.5-1 }}$ and $\lambda_{\text {BNF.5-2 }}$ are negative and positive, respectively, for all measured values, but Eqs. (45) and (46) indicate will vary with changes in $k_{10}$, especially those in the vicinity of the singularity in CP 5 that occurs when:

$k_{10}=k_{25}\left(1-k_{11}\right)=0.425$

However, CP 5 is infeasible for all $k_{10}>0.3973$ and CP 5 is a saddle point for most values of $k_{10} \cdot \lambda_{\text {BNF.5-1 }}$ is not sensitive to $k_{13}$ and is always negative, while $\lambda_{B N F-5-2}$ is negative when:

$k_{13}>k_{8}\left(1-k_{14}\right)\left[\frac{k_{25}\left(1-k_{11}\right)-k_{10}\left(1+k_{26}\right)}{k_{25}\left(1-k_{11}\right)\left(1+k_{9}\right)-k_{10}\left(1+k_{9}+k_{26}\right)}\right]$

$=1.798$.

CP 5 is therefore a saddle point for measured parameter values, but becomes a stable node when $k_{13}>1.798$.

Increases in $k_{10}$ precipitate a transcritical bifurcation in the BFN sub-model when CP 5 collides with $\mathrm{CP} 4$, and CP 5 becomes a stable node. This occurs when $B_{4}^{*}=B_{5}^{*}=0.0170$ and $\lambda_{\text {BNF.5-2 }}<0$ which, from Eqs. (40) and (45), and Eqs. (45) and (49), occurs when:

$k_{10}=\frac{k_{25}\left(1-k_{11}\right)\left[k_{8}\left(1-k_{14}\right)-k_{13}\left(1+k_{9}\right)\right]}{\left[k_{8}\left(1-k_{14}\right)-k_{13}\right]\left(1+k_{26}\right)-k_{9} k_{13}}=0.3969$.

At this point $\mathrm{CP} 4$ leaves the feasible region because $F_{4}^{*}=0 \quad 577$ which Eq. (41) confirms occurs when:

$k_{10}=k_{25}\left(1-k_{11}\right)\left(\frac{N_{4}^{*}}{N_{4}^{*}+k_{26}}\right)=0.3969$

A small further increase in $k_{10}$ precipitates a second transcritical bifurcation in the BFN sub-model when CP 5 collides with CP 1, and CP 1 becomes a stable node which, from Eqs. (37) and (45), occurs very close to the first bifurcation, at:

$k_{10}=\frac{k_{25}\left(1-k_{11}\right)}{1+k_{26}}=0.3973$.

At this point CP 5 leaves the feasible region when $B_{5}^{*}=0$ which, from Eq. (45), occurs for the same condition as given by Eq. (54). CP 1 is then the only critical point of the BFN sub-model in the feasible region, and remains stable for further increases in $k_{10}$.

Variations in $k_{13}$ have more interesting effects. Evaluations of Eq. (43) reveal that the first event to occur as $k_{13}$ is increased is that CP 4 changes from an unstable spiral to a stable spiral point in a Hopf bifurcation (confirmed by numerical inspection of the vanishing limit cycle about CP 4) when $k_{13}=1.653$. This stability property is maintained until $k_{13}=1.760$ when $\mathrm{CP}$ 4 changes again, this time from a stable spiral to a stable node. This property holds until CP 4 collides with CP 5 in a transcritical bifurcation when $F_{4}^{*}=0$ which from Eq. (41) occurs when:

and $B_{4}^{*}=B_{5}^{*}$ which from Eqs. (40) and (45) confirms occurs when:

$k_{13}=\frac{k_{8}\left(1-k_{14}\right)\left[k_{25}\left(1-k_{11}\right)-k_{10}\left(1+k_{26}\right)\right]}{k_{9}\left[k_{25}\left(1-k_{11}\right)-k_{10}\right]+k_{25}\left(1-k_{11}\right)-k_{10}\left(1+k_{26}\right)}=1.798$

At this point CP 5 changes from a saddle point to a stable node according to Eq. (51) and CP 4, previously a stable node, simultaneously becomes a saddle point and infeasible.

The direction field of the BFN sub-model for measured parameter values (Fig. 7) is characterised by the ubiquity of near horizontal flows pushing $B$ towards zero throughout the feasible state space, and the location of all critical points at or in the vicinity of $F=0$. This is due to the influence of the high maximum rate of $F$ predation on $B$. Once $B$ is very close to zero (within $10^{-3}$ ), $F$ decays and flows on the $F$ axis are vertically down (this is shown in the expanded region of the BF plane in Fig. 7). After $F$ has fallen to very low levels (again less than $10^{-3}$ ), $B$ can then grow and flows along the $B$ axis are directed towards CP 5 .

The dynamics of the BFN sub-model reveal an apparently closed stable orbit, to which initial conditions both inside and outside the orbit are attracted, bounded by CP 5 and CP 1 , and containing CP 4. This closed orbit, for measured parameter values, pushes trajectories we are interested in very close to $\mathrm{CP} 1$, and hence they spend a long time near CP 1 . Variations in $k_{13}$ lead to a Hopf bifurcation of this closed orbit at CP 4 , when CP 4 changes stability. .

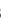
.

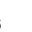

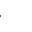

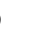
. . (1) 


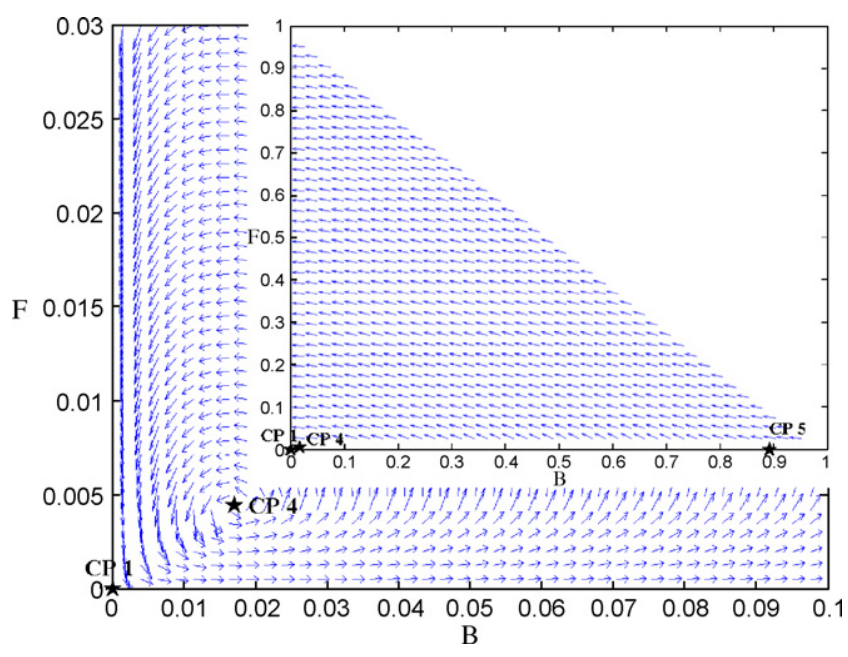

Fig. 7 - Direction field of the BFN model shown in BF state space. As $k_{10}$ increases CP 5 approaches CP 4, while as $k_{13}$ increases CP 4 approaches CP 5.

$652 \lambda \lambda_{\text {CP_1-1 }}=\frac{k_{23}}{1+k_{24}}$,

$653 \quad \lambda_{\mathrm{CP}_{-} 1-2}=\frac{k_{25}\left(1-k_{11}\right)}{1+k_{26}}-k_{10}$,
$\lambda_{\mathrm{CP}_{-1}-3}=-k_{19}$,

$\lambda_{\mathrm{CP} \_1-4}=-k_{13}$,

$\lambda_{\mathrm{CP}_{\text {_1-1 }}}$ is always positive, while $\lambda_{\mathrm{CP} \_1-3}$ and $\lambda_{\mathrm{CP}_{\text {_1 }} 4}$ are always negative. $\lambda_{\text {CP_1-2 }}$ is positive for measured parameter values, but is negative for:

$k_{10}>\frac{k_{25}\left(1-k_{11}\right)}{1+k_{26}}=0.397$.

CP 1 will therefore always be a saddle point in the GMSK model as it is in the sub-models.

The GMSK and NPZ models also share the autotroph-only critical point (CP 2):

$P_{2}^{*}=1$,

$B_{2}^{*}, F_{2}^{*}, N_{2}^{*}, Z_{2}^{*}=0$

Useful analytic expressions may be obtained for the eigenvalues of CP 2:

$\lambda_{C P \_2-1}=k_{4}\left(1-k_{20}\right)-k_{19}$,

$\lambda_{\mathrm{CP} \_2-2}=\frac{k_{1}\left(1-k_{11}\right)}{1+k_{2}}-k_{10}$,

$\lambda_{\mathrm{CP} \_2-3}=-\frac{k_{23}}{k_{24}}$

$\lambda_{\mathrm{CP} \_2-4}=-k_{13}$.

$\lambda_{\mathrm{CP} \_2-3}$ and $\lambda_{\mathrm{CP} \_-4}$ are always negative, while $\lambda_{\mathrm{CP} \_2-1}$ is positive and $\lambda_{\mathrm{CP} 2-2}$ negative for measured parameter values, but may change sign depending on the values of $k_{10}$ and $k_{13} \cdot \lambda_{\text {CP_2- } 1}$ is negative when:

$k_{19}>k_{4}\left(1-k_{20}\right)=1.111$

while $\lambda_{\text {CP_2-2 }}$ is positive when:

$k_{10}<\frac{k_{1}\left(1-k_{11}\right)}{1+k_{2}}=0.251$

CP 2 may therefore be a saddle point or a stable node for the GMSK model as it is for the NPZ sub-model.

A third critical point (CP 3) is also shared by the GMSK and NPZ models, and is given by:

$P_{3}^{*}=\frac{k_{19}}{k_{4}\left(1-k_{20}\right)}$,

$Z_{3}^{*}=\frac{k_{23}}{k_{4}}\left(\frac{N_{3}^{*}}{N_{3}^{*}+k_{24}}\right)$,

68

$N_{3}^{*}=\frac{1}{2}\left[-\left(k_{24}+P_{3}^{*}+\frac{k_{23}}{k_{4}}-1\right)\right.$

$\left.\pm \sqrt{\left(k_{24}+P_{3}^{*}+\frac{k_{23}}{k_{4}}-1\right)^{2}-4 k_{24}\left(P_{3}^{*}-1\right)}\right]$,

654

655

656 657 658

660 661 662 663 664 665 666 667 669 670 671 672 673 674 675 
$B_{3}^{*}, F_{3}^{*}=0$

Analytic expressions for the eigenvalues of this model at CP 3 are not useful, and these eigenvalues will be calculated numerically. The fourth critical point of the GMSK model (CP 4) is shared with the BFN sub-model and is located at:

$B_{4}^{*}=\frac{k_{9} k_{13}}{k_{8}\left(1-k_{14}\right)-k_{13}}$,

$F_{4}^{*}=\frac{k_{25}}{k_{8}}\left(1-k_{11}\right)\left(B_{4}^{*}+k_{9}\right)\left(\frac{N_{4}^{*}}{N_{4}^{*}+k_{26}}\right)-\frac{k_{10}}{k_{8}}\left(B_{4}^{*}+k_{9}\right)$,

$N_{4}^{*}=\frac{1}{2}\left[-\left(k_{26}+B_{4}^{*}+\left(\frac{B_{4}^{*}+k_{9}}{k_{8}}\right)\left(k_{25}\left(1-k_{11}\right)-k_{10}\right)-1\right)\right.$
$P_{4}^{*}, Z_{4}^{*}=0$.

Similarly, analytic expressions for the eigenvalues at $\mathrm{CP} 4$ are not useful, and these eigenvalues will also be calculated numerically. The fifth critical point of the GMSK model (CP 5) is also shared with the BFN sub-model and is given by:

$B_{5}^{*}=1-\frac{k_{10} k_{26}}{k_{25}\left(1-k_{11}\right)-k_{10}}$,

$N_{5}^{*}=\frac{k_{10} k_{26}}{k_{25}\left(1-k_{11}\right)-k_{10}}$

$F_{5}^{*}, P_{5}^{*}, Z_{5}^{*}=0$.

Useful expressions for two of the eigenvalues of CP 5 are obtainable:

$\lambda_{\text {CP }-5-1}=k_{23}\left(\frac{N_{5}^{*}}{N_{5}^{*}+k_{24}}\right)-\left(\frac{k_{1}}{k_{2}}\right) B_{5}^{*}$,

$\lambda_{\mathrm{CP} \_5-2}=-k_{19}$,

while the other two are given by:

$\lambda_{\mathrm{CP} \_5-3,4}=\frac{\omega_{5}+\xi_{5} \pm \sqrt{\left(\omega_{5}-\xi_{5}\right)^{2}+4 \psi_{5} \zeta_{5}}}{2}$

where

$\omega_{5}=-k_{25}\left(1-k_{11}\right)\left(\frac{k_{26}}{\left(N_{5}^{*}+k_{26}\right)^{2}}\right) B_{5}^{*}-k_{8} k_{14}\left(\frac{B_{5}^{*}}{B_{5}^{*}+k_{9}}\right)-k_{13}$,

$\xi_{5}=k_{25}\left(1-k_{11}\right)\left(\frac{N_{5}^{*}}{N_{5}^{*}+k_{26}}\right) B_{5}^{*}+k_{8}\left(\frac{B_{5}^{*}}{B_{5}^{*}+k_{9}}\right)-k_{10}$,

$\psi_{5}=k_{10}-k_{25}\left(1-k_{11}\right)\left(\frac{N_{5}^{*}}{N_{5}^{*}+k_{26}}\right)-k_{8} k_{14}\left(\frac{B_{5}^{*}}{B_{5}^{*}+k_{9}}\right)-k_{13}$,
$\zeta_{5}=k_{25}\left(1-k_{11}\right)\left(\frac{k_{26}}{\left(N_{5}^{*}+k_{26}\right)^{2}}\right) B_{5}^{*}+k_{8}\left(\frac{B_{5}^{*}}{B_{5}^{*}+k_{9}}\right)$.

$\lambda_{\text {CP.5-2 }}$ is always negative, and $\lambda_{\text {CP.5-1 }}$ is negative for measured parameter values. Numerical evaluations reveal that $\lambda_{\text {CP. } 5-1}$ is insensitive to variations in $k_{13}$ and $k_{19}$, but becomes positive when $k_{10}>0.379$. Numerical evaluation of Eq. (85) reveals that $\lambda_{\text {CP.5-3 }}$ and $\lambda_{\text {CP. } 5-4}$ are real and have one positive and one negative value for all values of $k_{10}$ used in this analysis, but have two negative real values for $k_{13}>1.798 . \lambda_{\mathrm{CP} .5-3}$ and $\lambda_{\mathrm{CP} .5-4}$ are insensitive to variations in $k_{19}$. This reproduces the BFN submodel behaviour for CP 5 .

The GMSK model has a further two critical points, that are not shared with the sub-models. The first (CP 6) is where four

719 720

$P_{6}^{*}=\frac{k_{1}}{k_{23}}\left(\frac{N+k_{24}}{N}\right) B_{6}^{*}-k_{2}$,

$B_{6}^{*}=\frac{k_{9} k_{13}}{k_{8}\left(1-k_{14}\right)-k_{13}}$,

$F_{6}^{*}=\frac{k_{25}}{k_{8}}\left(1-k_{11}\right)\left(B_{6}^{*}+k_{9}\right)\left(\frac{N_{6}^{*}}{N_{6}^{*}+k_{26}}\right)-\frac{k_{10}}{k_{8}}\left(B_{6}^{*}+k_{9}\right)$,

$Z_{6}^{*}=0$,

$N_{6}^{* 4}+N_{6}^{* 3}\left[k_{26}+k_{24}-1-k_{2}+B_{6}^{*}-\beta_{6} k_{10}+\alpha_{6}\right.$

$\left.+\beta_{6} \gamma_{6}\left(1-\frac{k_{2}}{\alpha_{6}}\right)+\beta_{6} \delta_{6}\right]+N_{6}^{* 2}\left[k_{26}\left(k_{24}-1-k_{2}+B_{6}^{*}\right.\right.$$$
\left.-\beta_{6} k_{10}+\alpha_{6}+\beta_{6} \gamma_{6}\left(1-\frac{k_{2}}{\alpha_{6}}\right)\right)+\alpha_{6} k_{24}-k_{24}\left(1+k_{2}-B_{6}^{*}\right.
$$

$\left.\left.+\beta_{6} k_{10}-\alpha_{6}\right)+\beta_{6} k_{24}\left(\delta_{6}+\gamma_{6}\right)\right]+N_{6}^{*}\left[\alpha_{6} k_{24}^{2}\right.$

$\left.+k_{26}\left(\alpha_{6} k_{24}-k_{24}\left[1+k_{2}-B_{6}^{*}+\beta_{6} k_{10}-\alpha_{6}\right]+\beta_{6} \gamma_{6} k_{24}\right)\right]$

$+\alpha_{6} k_{24}^{2} k_{26}=0$

where $\alpha_{6}=k_{1} B_{6}^{*} / k_{23}, \quad \beta_{6}=B_{6}^{*}+k_{9} / k_{8}, \quad \delta_{6}=k_{25}\left(1-k_{11}\right), \quad \varepsilon_{6}=$ $k_{2} k_{23} / k_{1} B_{6}^{*}$ and $\gamma_{6}=k_{1}\left(1-k_{11}\right)$ and only the positive roots are considered.

Again, analytic expressions for the eigenvalues at CP 6 are not useful, and these eigenvalues will also be calculated numerically. The second (and final) critical point of the GMSK model (CP 7), that is not shared by the sub-models is the only critical point to have all positive, non-zero state variables such that:

$P_{7}^{*}=\frac{k_{19}}{k_{4}\left(1-k_{20}\right)}$,

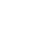

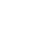


$753 \quad B_{7}^{*}=\frac{k_{9} k_{13}}{k_{8}\left(1-k_{14}\right)-k_{13}}$,

754

755

$$
\begin{aligned}
F_{7}^{*}= & \left(\frac{B_{7}^{*}+k_{9}}{k_{8}}\right)\left[k_{1}\left(1-k_{11}\right)\left(\frac{P_{7}^{*}}{P_{7}^{*}+k_{2}}\right)\right. \\
& \left.+k_{25}\left(1-k_{11}\right)\left(\frac{N_{7}^{*}}{N_{7}^{*}+k_{26}}\right)-k_{10}\right],
\end{aligned}
$$

$$
Z_{7}^{*}=\left(\frac{k_{23}}{k_{4}}\right)\left(\frac{N_{7}^{*}}{N_{7}^{*}+k_{24}}\right)-\left(\frac{k_{1}}{k_{4}}\right)\left(\frac{B_{7}^{*}}{P_{7}^{*}+k_{2}}\right)
$$

$$
\begin{aligned}
N_{7}^{* 3} & +N_{7}^{* 2}\left[P_{7}^{*}+B_{7}^{*}+\alpha_{7}+\beta_{7}+\delta_{7}+k_{24}+k_{26}-\gamma_{7}-\varepsilon_{7}-1\right] \\
& +N_{7}^{*}\left[k_{24} k_{26}+\left(k_{24}+k_{26}\right)\left(P_{7}^{*}+B_{7}^{*}+\alpha_{7}-\gamma_{7}-\varepsilon_{7}-1\right)\right. \\
& \left.+k_{24} \beta+k_{26} \delta_{7}\right]+k_{24} k_{26}\left(P_{7}^{*}+B_{7}^{*}+\alpha_{7}-\gamma_{7}-\varepsilon_{7}-1\right)=0,
\end{aligned}
$$

where $\alpha_{7}=k_{1}\left(1-k_{11}\right)\left(P_{7}^{*} / P_{7}^{*}+k_{2}\right)\left(B_{7}^{*}+k_{9} / k_{8}\right), \beta_{7}=k_{25}\left(1-k_{11}\right)$ $\left(B_{7}^{*}+k_{9} / k_{8}\right), \quad \gamma_{7}=k_{10}\left(B_{7}^{*}+k_{9} / k_{8}\right), \quad \delta_{7}=k_{23} / k_{4} \quad$ and $\quad \varepsilon_{7}=$ $\left(k_{1} / k_{4}\right)\left(B_{7}^{*} / P_{7}^{*}+k_{2}\right)$ and only the positive root is considered.

As for the critical points CP 3, CP 4 and CP 6 of the GMSK model, analytic expressions for the eigenvalues at CP 7 are not useful, and these eigenvalues will be calculated numerically.

The equations above describe the seven critical points of the GMSK model that are located in the ecologically-feasible $(0 \leq B, F, N, P, Z \leq 1)$, mass conservative $(B+F+N+P+Z=1)$ region of state space for the measured parameter values. A further seven critical points described by these equations are located in ecologically unrealistic regions of the state space and are not considered. The locations of the seven feasible critical points for measured parameter values are given in Table 2, and are shown in Figs. 2 and 3. These critical points appear to substantially control the shape of the GMSK model's limit cycle.

Table 2 and Figs. 2 and 3 reveal that the seven feasible critical points of the model form three pairs of closely aligned points: CP 3 and 7 lie very close, as do CP 1 and 4, and CP 2 and 6. CP 5 is unique in the GMSK model in that it is a lone feasible critical point for measured parameter values. The locations of most of the critical points can, however, be expected to change in response to variations in the parameters of interest, the mortality rates of $B, F$ and $Z, k_{10}, k_{13}$ and $k_{19}$. Eqs. (57), (58), (64) and (65) reveal that the locations of CP 1 and CP 2 are not affected by changes in $k_{10}, k_{13}$ or $k_{19}$; Eqs. (72)-(75) indicate that CP 3 is sensitive only to $k_{19}$; Eqs. (80)-(82) indicate CP 5 is sensitive to $k_{10}$ only; Eqs. (76)-(79), (86) and (90) indicate CP 4 and CP 6 are sensitive to $k_{10}$ and $k_{13}$ and Eqs. (91)-(95) reveal the position of CP 7 is sensitive to changes in $k_{10}, k_{13}$ and $k_{19}$.

\subsubsection{Numerical eigenvalues and eigenvectors}

Numerical evaluation of the eigenvalues of the critical points for measured parameter values reveals that all are unstable, with each critical point having at least one eigenvalue with a positive real part. All the critical points of the GMSK model are therefore higher order saddles and spirals or nodes for measured parameter values, with some positive and some negative eigenvalues. The nature of the system dynamics in the regions near the critical points varies depending on the number of real and complex eigenvalues each point has, and the direction in which the associated eigenvectors point. Values for the numerically calculated eigenvalues of each of the critical points for the measured parameter values are given in Table 3, and the numerically calculated eigenvectors in Table 4.

Eigenvectors of selected large magnitude positive (outward pointing) and negative (inward pointing) eigenvalues are shown for each critical point in Figs. 2 and 3. The eigenvectors at each critical point were calculated numerically from the five-equation system to enable the eigenvectors to be plotted in both NPZ and BFN spaces (this calculation always resulted in one zero eigenvalue, which is ignored). The lengths of the eigenvectors have been approximately scaled by the largest magnitude eigenvalue at each critical point.

The importance of the critical points $\mathrm{CP} 3$ and $\mathrm{CP} 7$ is apparent in Fig. 2. CP 3 is located near the centre of the spiral in the NPZ simplex plane, with CP 7 nearby just off the simplex plane. Interestingly, it is the critical point $\mathrm{CP} 3$ (with $B_{3}^{*}, F_{3}^{*}=0$ ) that controls the dynamics of the GMSK model, rather than the critical point CP 7 that has all species extant. Although the dominant eigenvectors of both $\mathrm{CP} 3$ and $\mathrm{CP} 7$ direct the flow off the NPZ simplex plane and towards the origin of the NPZ space, the dominant eigenvalue of CP 3 is two orders of magnitude larger than the real part of the dominant eigenvalue of $\mathrm{CP} 7$, and the flow is observed to leave the simplex plane along the dominant eigenvector of $\mathrm{CP} 3$ rather than that of $\mathrm{CP}$ 7. However, we note that the system is never actually on the simplex planes as $B$ and $F$ in the case of the NPZ plane, and $P$ and $Z$ in the case of the BFN plane (Fig. 3) are very small but never exactly zero; for ease of discussion we will not labour this distinction.

The dominant eigenvector of CP 3 in BFN space directs the system towards CP 5 and 'onto' the BFN simplex plane. The trajectory is captured by the attracting eigenvector of $\mathrm{CP} 5$ and then directed on to CP 1 and CP 4 by its repelling eigenvector. Fig. 3 reveals that this eigenvector directs the flow approximately orthogonally to the attracting eigenvectors of CP 1 and $\mathrm{CP} 4$ giving the appearance that a further critical point lies at approximately $F=0.3, N=0.7, P, B, Z=0$. However, this is not the case as $B$ has a large negative flux and $F$ and $N$ have large positive fluxes in this region, indicating that the dynamics are controlled by the eigenvectors of CP 1, CP 4 and CP 5 and the mass conservation boundary.

\subsection{Bifurcation in the GMSK model}

We preface our analysis of the bifurcation behaviour of the GMSK system in four dimensions by noting that it does not exhibit the "clean" behaviour observed in the two-dimensional systems. As we are evaluating many of the eigenvalues numerically, this raises the possibility of programming errors affecting our observed behaviours. However, we can confirm the correct programming of the analytic expressions for the critical points by checking that they conserve mass, which all critical points do to machine accuracy $\left(10^{-14}\right)$. The numerically calculated eigenvalues were checked by comparing with analytic eigenvalues where they 

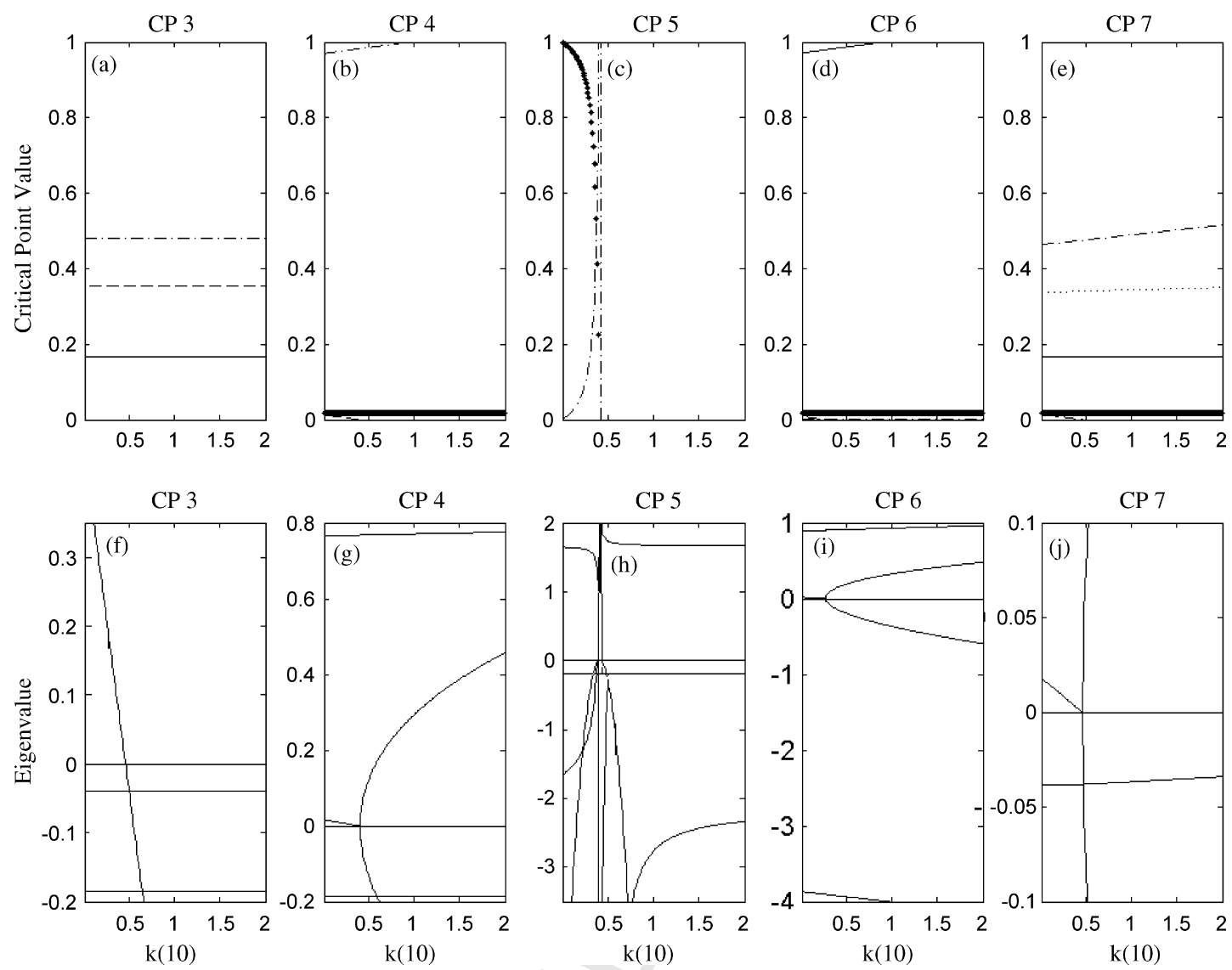

Fig. 8 - Selected critical points and associated real parts of eigenvalues for the GMSK model for variations in $k_{10}$. In the critical point plots, $B$ is represented by the thick dotted line, $F$ the dashed line, $N$ the dot-dash line, $P$ the solid line and $Z$ the dotted line.

where $B_{4}^{*}=B_{5}^{*}=k_{9} k_{13} / k_{8}\left(1-k_{14}\right)-k_{13}$. A "transcritical" bifurbehaviours to future research. We have elected to refer to the behaviours we observe in the GMSK model according to the two-dimensional behaviour most closely related to each and to note, but not labour, the differences.

\subsubsection{Variations in bacteria mortality $\left(k_{10}\right)$}

We have previously noted that the locations of critical points CP 3, 4, 5, 6 and 7 are sensitive to variations in $k_{10}$. The responses of these critical points and the real parts of their eigenvalues to variations in $k_{10}$ are shown in Fig. 8 . This reveals that critical points CP 2, 3, 6 and 7 lose feasibility as $k_{10}$ increases, and that $\mathrm{CP} 3$ eventually becomes stable. As $k_{10}$ increases from its measured value CP 5 approaches CP 4 and $F_{4}^{*}$ slowly reduces until the two critical points collide when $F_{4}^{*}$ becomes zero which, from Eq. (77), occurs when:

$k_{10}=k_{25}\left(1-k_{11}\right)\left(\frac{1-B_{4}^{*}}{1-B_{4}^{*}+k_{26}}\right)=0.3969$, cation occurs at this point, although no transfer of stability takes place as both critical points are unstable before and after the collision. CP 4 is now infeasible and as $k_{10}$ is increased further $\mathrm{CP} 5$ also becomes infeasible when $B_{5}^{*}$ becomes one, which from Eq. (80), occurs when:

$k_{10}=\frac{k_{25}\left(1-k_{11}\right)}{1+k_{26}}=0.3973$.

This is the same condition for which the eigenvalue $\lambda_{B F N-1-1}$ of CP 1 (Eq. (39)) becomes negative in the BFN sub-model, and $\lambda_{\text {CP-1-2 }}$ of CP 1 (Eq. (60)) becomes negative in the GMSK model.

Up to this point the bifurcation behaviour of the BFN submodel and the GMSK model with respect to $k_{10}$ are identical. However, in the GMSK model the eigenvalue $\lambda_{\text {CP.1-1 }}$ of CP 1 is never negative, so when CP 1 and CP 5 collide CP 1 does not become a stable node as it does in the BFN sub-model.

The exclusion of CP 4 and CP 5 from the feasible orthant removes the system's initial and second attracting points on the BFN plane (Fig. 3). The repelling eigenvector of CP 7 (Fig. 2) still directs the flow off the NPZ plane towards the BFN plane, resulting in a B bloom. However now the only influences on the system are the attracting eigenvectors of $\mathrm{CP} 1$, and the brief $B$ bloom collapses without generating a subsequent $F$ bloom. Whilst modifying the shape of the attractor as the system now no longer reaches the BFN plane; this does not, however, result 

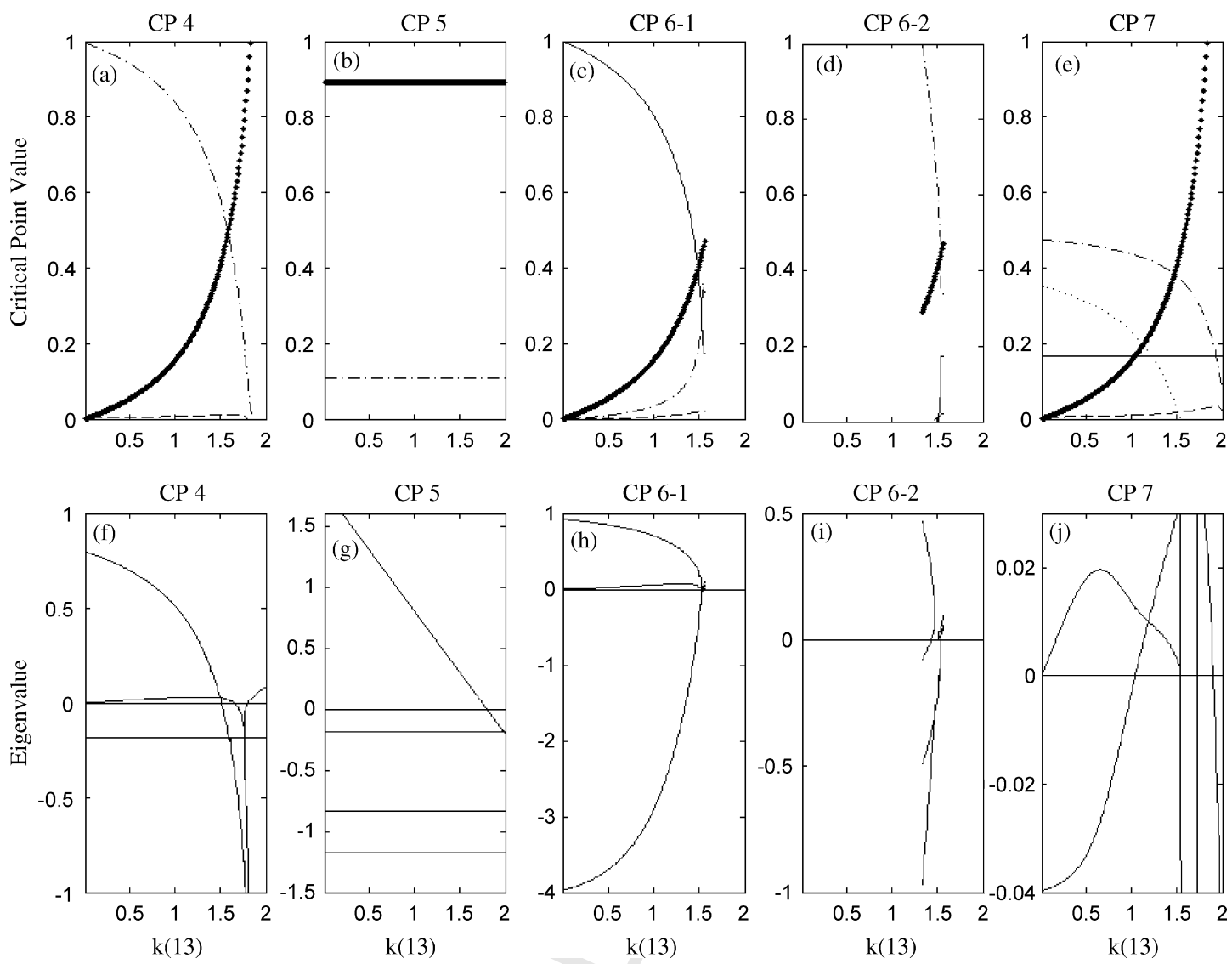

Fig. 9 - Selected critical points and associated real parts of eigenvalues for the GMSK model for variations in $k_{13}$.

in a markedly different time series from those presented in Fig. 4.

If $k_{10}$ exceeds the value defined by Eq. (97) the system is in Mode A most of the time, taking short excursions into the reduced Mode $B$. However when:

$k_{10}=k_{1}\left(1-k_{11}\right)\left(\frac{P_{7}^{*}}{P_{7}^{*}+k_{2}}\right)+k_{25}\left(1-k_{11}\right)\left(\frac{N_{7}^{*}}{N_{7}^{*}+k_{26}}\right)=0.453$,

$F_{7}^{*}$ becomes zero, and CP 3 and CP 7 "collide" (they never actually collide as $B_{7}^{*}$ is near, but never equals zero, so $Z_{3}^{*}$ never equals $Z_{7}^{*}$ and $N_{3}^{*}$ never equals $N_{7}^{*}$ ). At this point a bifurcation occurs in CP 3 as the system changes from a limit cycle to an asymptotically stable node at CP 3. This is not a Hopf bifurcation as the real parts of the complex eigenvalues of CP 3 do not pass through zero - stability is conferred by the single positive (real) eigenvalue of CP 3 becoming negative (Fig. 8f). This state then holds for all further increases in $k_{10}$.

When $k_{10}$ exceeds the value specified in Eq. (98) we can see from Eq. (2) that $d B / d t$ is always negative when the system is in the vicinity of CP 7 . The system is now prevented from flipping to the BFN plane and is permanently in Mode A. Increases in B mortality can therefore fundamentally change the dynamics of the system. Eq. (98) reflects a balance between bacterial growth (expressed as a function of maximum potential growth rates, assimilation efficiency and food availability) and mortality. The model therefore suggests the interesting ecological speculation that low nutrient plankton systems will be less susceptible to bacterial perturbation than high nutrient systems. This is due to increases in the total nutrient having the effect of increasing the natural bacterial mortality rate required to preclude bacterial blooms. This suggests that the addition of nutrients to otherwise stable plankton ecosystems (as humans invariably do with sewerage outfalls and agricultural run-off) could fundamentally change the dynamics of those ecosystems from plankton-controlled to microbecontrolled.

\subsubsection{Variations in zooflagellate mortality $\left(k_{13}\right)$}

We noted above that variations in $k_{13}$ have a similar (but inverse) effect on the switching behaviour to variations in $k_{10}$; however, the bifurcation effects of these two parameters are quite different. Whereas increases in $k_{10}$ quite quickly push the system into a permanent "Mode A steady state" at CP 3, increases in $k_{13}$ do not precipitate dramatic changes in the system until $k_{13}$ becomes very large.

The locations of critical points CP 4, 6 and 7 are sensitive to variations in $k_{13}$ (note that CP 6 has two feasible roots for some values of $k_{13}$ ). The location of CP 5 is insensitive to variations in $k_{13}$ but the eigenvalues of this point are affected by variations in the parameter. The responses of these critical points and the real parts of their eigenvalues to variations in $k_{13}$ are shown in Fig. 9. This reveals that critical points $C P 4,6$ and 7 all become infeasible as $k_{13}$ increases. 
The first change that occurs as $k_{13}$ increases is apparent in Fig. 9d where, when $k_{13}=1.508$, a second root of Eq. (90), CP $6-2$, becomes feasible. Eq. (90) has two positive roots for values of $k_{13}$ less than 1.508 , but the second root only becomes ecologically feasible when $\mathrm{F}_{6-2}^{*}$ becomes positive after $\mathrm{N}_{6-2}^{*}$ has become less than one. This second critical point however soon disappears along with CP 6-1 in a saddle-node bifurcation when $k_{13}=1.537$. This change in CP 6 coincides with the loss of feasibility of CP 7 that occurs when $Z_{7}^{*}=0$, which Eqs. (91), (92) and (94) indicate occurs when:

$k_{13}=\frac{k_{8} k_{23}\left(1-k_{14}\right)\left(\left(k_{19} / k_{4}\left(1-k_{20}\right)\right)+k_{2}\right)}{k_{1} k_{9}\left(1+\left(k_{24} / N_{7}^{*}\right)\right)+k_{24}\left(\left(k_{19} / k_{4}\left(1-k_{20}\right)\right)+k_{2}\right)}=1.537$

These events are not accompanied by any change in the stability properties of the system. However as $k_{13}$ approaches this value the magnitude of the $P$ blooms decreases and the system progressively spends more time in Mode B until at $k_{13}=1.537$ it appears that $P$ no longer blooms (to machine resolution). The system no longer visits Mode $A$ and is effectively confined to the BFN limit cycle characteristic of Mode B.

As $k_{13}$ increases further the real parts of the eigenvalues of CP 4 all become negative (Fig. 9f) when $k_{13}=1.653$. At this point CP 4 becomes a stable spiral node in a Hopf bifurcation. Numerical integration of the system confirms that the limit cycle surrounding CP 4 shrinks as $k_{13}$ increases and vanishes when $k_{13}=1.653$, as was observed for the BFN sub-model. CP 4 remains stable until it collides with $\mathrm{CP} 5$ in a transcritical bifurcation that occurs when $F_{4}^{*}=0$ that Eq. (77) indicates occurs at:

$k_{13}=k_{8}\left(1-k_{14}\right)\left(\frac{B_{4}^{*}}{B_{4}^{*}+k_{9}}\right)=1.798$,

where $B_{4}^{*}=B_{5}^{*}=1-\left(k_{10} k_{26}\right) /\left(k_{25}\left(1-k_{11}\right)-k_{10}\right)=0.8919$. At this point $\mathrm{CP} 4$ becomes a saddle point, and then becomes infeasible when $B_{4}^{*}=1$ at:

$k_{13}=\frac{k_{8}\left(1-k_{14}\right)}{1+k_{9}}=1.831$,

while CP 5 acquires stability and remains a stable node for further increases in $k_{13}$. This bifurcation behaviour for the GMSK model is therefore identical to that of the BFN sub-model.

The results of increasing $k_{13}$ have a straightforward, intuitively obvious and well-accepted ecological interpretation: if the mortality of a predator is increased to the point at which a population can no longer survive in an ecosystem, its prey will come to dominate the ecosystem.

\subsubsection{Variations in zooplankton mortality $\left(k_{19}\right)$}

Increases in $k_{19}$ do not precipitate any qualitative changes in the critical points until $k_{19}$ becomes quite large; however, we
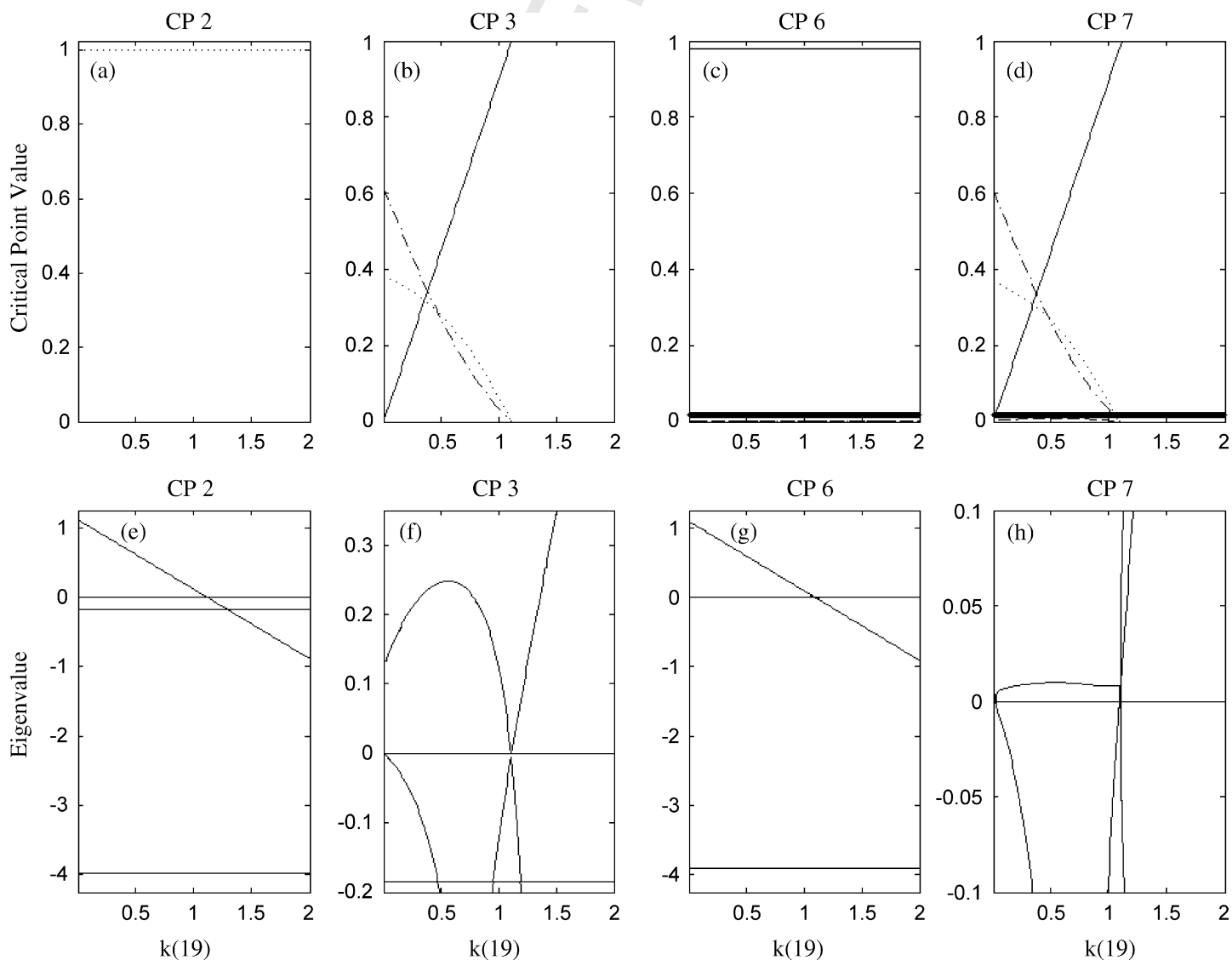

Fig. 10 - Selected critical points and associated real parts of eigenvalues for the GMSK model for variations in $k_{19}$. [Note: (g) includes a constant eigenvalue at $+\mathbf{0 . 0 0 7 5}$. 
note that at $k_{19}=0.790$ two of the eigenvalues of CP 7 become real and at $k_{19}=0.810$ all the eigenvalues of CP 3 become real. Although two eigenvalues of CP 7 remain complex, the oscillatory dynamics that were previously an enduring characteristic of the NPZ part of the GMSK attractor are lost.

Further increases in $k_{19}$ engender a transcritical bifurcation when:

$k_{19}=k_{4}\left(1-k_{20}\right)=1.111$

at which point CP 3 collides, and CP 7 "almost collides", with CP 2 ( $B_{7}^{*}$ is not affected by $k_{19}$ and remains non-zero and not equal to $B_{2}^{*}$, so $Z_{7}^{*}$ never equals $Z_{2}^{*}$ and $N_{7}^{*}$ never equals $N_{2}^{*}$ ). When this occurs the single positive eigenvalue of CP $2\left(\lambda_{\mathrm{CP}-2-1}\right)$ passes through zero to become negative (Eq. (66) and Fig. 10e) and CP 2 becomes an asymptotically stable node. This behaviour is analogous to the transcritical bifurcation observed in the NPZ sub-model. CP 3 and 7 remain unstable and leave the feasible region at this point as $P_{2}^{*}>1$ (Fig. $10 b$ and d). This behaviour has attributes of a transcritical bifurcation, but in this case the system has changed from a limit cycle to a stable node. As noted above, all the eigenvalues of the system have become real before this point, and the change in stability is not the result of Hopf bifurcation.

We also note that CP 6 is very close to CP 2 , and exhibits similar eigenvalue behaviour as CP 2 (Fig. 10a, c, e and g), although one eigenvalue of CP 6 always remains slightly positive. As the eigenvalues of CP 2 are linearly related to $k_{19}$, this point remains stable as $k_{19}$ is further increased (Eqs. (66)-(69) and Fig. 10e). The bifurcation behaviours of the NPZ sub-model and the GMSK model occur at the same point and are very similar, both undergoing transcritical bifurcations when $k_{19}=1.111$. The only difference is that rather than CP 3 changing from a stable spiral to a saddle, as it does in the NPZ sub-model, in the GMSK model CP 3, which changes from an unstable spi- ral to an asymptotically unstable node as $k_{19}$ increases from its measured value, remains an asymptotically unstable node throughout the bifurcation. Further, CP 3's movement through the NPZ state space is mirrored by CP 7, which does not technically take part in the transcritical bifurcation although its eigenvalues change at the bifurcation point (but its stability properties do not).

It is readily apparent that increases in $k_{19}$ have the same ecological interpretation as increases in $k_{13}$ : if the predator can no longer survive in an ecosystem, the prey will come to dominate. When $k_{13}$ was increased, the system became dominated by bacteria; when $k_{19}$ was increased, the system became dominated by phytoplankton, despite the fact that $P$ was still predated on by $B$.

3.6.4. Simultaneous variations in all mortality parameters Simultaneous variation in $k_{10}, k_{13}$ and $k_{19}$ describes a climate change scenario in which all species are similarly affected by changes in temperature. This is a reasonable scenario given that most species in an ecosystem will be adapted to the ambient temperatures associated with the previous climate if it has been stable for some time. Species affected by climate change may experience increases in their mortality rates due to environmental stresses, or reductions in mortality due to environmental stresses affecting their predators. While both increases and decreases of mortality rates have been investigated here, it is the increases that lead to the most interesting changes in the models. Simultaneous uniform variation in $k_{10}, k_{13}$ and $k_{19}$ leads to similar bifurcation behaviours to those described above. The locations of some bifurcation points move slightly in the state space due to the influence of more than one parameter, and the parameter values at which some critical points lose ecological feasibility also move for the same reason. The salient effect of varying all the mortality parameters together is that the critical points CP 5
1034 1035 1036 1037 1038 1039 1040 1041 1042 1043 1044 1045 1046 1047

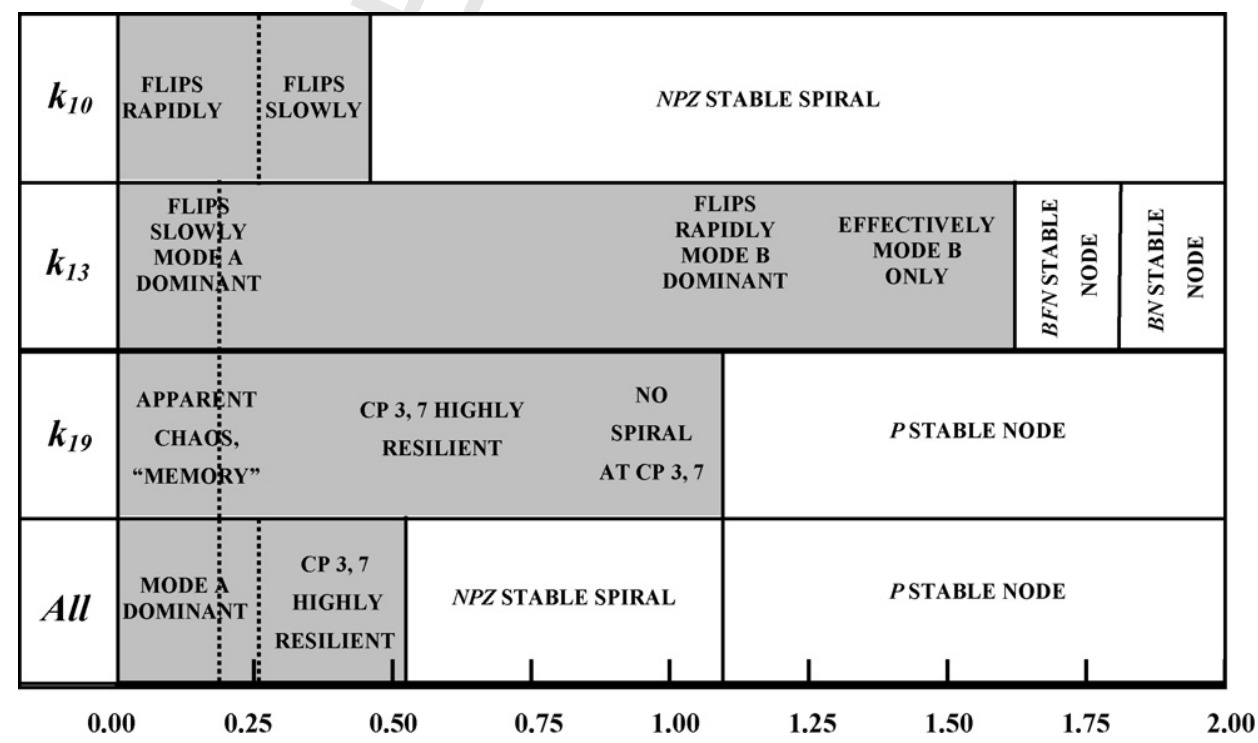

Fig. 11 - Schematic of the dynamical behaviour of the GMSK model in response to one-at-a-time parameter variations. The grey region indicates extent of the characteristic Mode A/Mode B dynamics with subtle modifications noted. Dotted lines denote measured values. 
and 3, that previously became stable for perturbations of $k_{10}$ singly, no longer do so. The only critical points that become stable are CP 3, which now becomes stable at 0.506 , and CP $2\left(P_{2}^{*}=1\right)$, which acquires stability at 1.111 , the same value as previously observed for the bifurcation in the GMSK model and the NPZ sub-model. This bifurcation point is unaffected by the simultaneous variations in $k_{10}$ and $k_{13}$. At this point also the eigenvalues of all the critical points all become real. For mortality parameter values greater than 1.111, the only other ecologically feasible critical point is CP $1\left(N_{1}^{*}=1\right)$. This critical point has one positive eigenvalue $\left(\lambda_{\mathrm{CP}_{-} 1-1}=k_{23} / 1+k_{24}\right)$ that is insensitive to all mortality parameter perturbations, indicating that CP 1 is always unstable.

Fig. 11 summarises the results of the bifurcation analysis, and demonstrates the robustness of the system's limit cycle dynamics to variations in $k_{10}, k_{13}$ or $k_{19}$. The mortality parameters generally require substantial variations in value before fundamentally affecting the dynamics. The $B$ mortality $\left(k_{10}\right)$ causes the earliest change, with a bifurcation from the limit cycle to a stable spiral at 1.75 times its measured value. When all parameters are varied together, this bifurcation occurs at a slightly higher value of $k_{10}$ (1.96 times its measured value). The $Z$ mortality $\left(k_{19}\right)$ is the next most sensitive parameter, with a similar bifurcation from the limit cycle to a stable spiral occurring at six times its measured value. The location of this bifurcation point is unaffected by variations in the other mortality parameters. The limit cycle dynamics are relatively insensitive to variations in the $F$ mortality $\left(k_{13}\right)$, with the first bifurcation occurring at nine times the measured value, and the second at approximately 10 times. Unlike the bifurcations due to $k_{10}$ and $k_{19}$ these bifurcations do not occur when all mortality parameters are varied simultaneously.

\subsubsection{An amended model}

The stability scenario described above raises an interesting question, for a simultaneous increase in mortality parameters is exactly the circumstance that ensues when ecosystems are subject to climate change, and species are pushed to the limits of their thermal ecological niches and beyond. However, our result that predicts an outcome where $P$ is the only survivor appears to be a result of the model formulation not including an explicit mortality term for $P$. This is often implicitly included by assuming that the maximum $P$ growth rate has been reduced by P's natural mortality. This is usually a reasonable assumption, however in the case of $\mathrm{CP} 2$, where $P$ consumes all available nutrient, the nutrient dependent growth rate approaches zero as $P$ approaches one. This suggests that, either both the reproduction and mortality rates have become zero, or that they are non-zero but all $P$ mortalities are instantaneously remineralised and up-taken by the still-growing $P$. Neither of these assumptions are valid, and the implicit representation of $P$ mortality is no longer reasonable.

Amending the GMSK model by including an explicit linear mortality term for $P$ in Eqs. (1) and (5):

$\frac{d P}{d t}=k_{23}\left(\frac{N}{N+k_{24}}\right) P-k_{1}\left(\frac{P}{P+k_{2}}\right) B-k_{4} P Z-k_{5} P$,

$$
\begin{aligned}
\frac{\mathrm{dN}}{\mathrm{dt}}= & k_{10} B+k_{11}\left[k_{25}\left(\frac{N}{N+k_{26}}\right) B+k_{1}\left(\frac{P}{P+k_{2}}\right) B\right] \\
& +k_{13} F+k_{8} k_{14}\left(\frac{B}{B+k_{9}}\right) F+k_{19} Z+k_{4} k_{20} P Z \\
& -k_{23}\left(\frac{N}{N+k_{24}}\right) P-k_{25}\left(\frac{N}{N+k_{26}}\right) B+k_{5} P,
\end{aligned}
$$

and considering the equivalent of CP 2 of the GMSK model in the new model (CP 2alt), we find that this critical point has become:

$P_{2}^{* *}=\frac{k_{23}-k_{5}\left(1+k_{24}\right)}{k_{23}-k_{5}}$,

$N_{2}^{* *}=1-\frac{k_{23}-k_{5}\left(1+k_{24}\right)}{k_{23}-k_{5}}$,

$B_{2}^{* *}, F_{2}^{* *}, Z_{2}^{* *}=0$.

When $k_{5}=0$ Eqs. (105)-(107) describe the same point as CP 2 of 1134 the GMSK model. The eigenvalues of this point are:

$\lambda_{\mathrm{CP}-2 \mathrm{alt}-1}=k_{4}\left(1-k_{20}\right)-k_{19}$

$\lambda_{\text {CP_2alt-2 }}=\frac{k_{1}\left(1-k_{11}\right)}{1+k_{2}}-k_{10}$,

$\lambda_{\text {CP_2alt }-3,4}=-\left(k_{5}+k_{13}+\frac{k_{23}}{k_{24}}\right)$

$$
\times\left(1 \pm \sqrt{1-\frac{4 k_{13} k_{23} k_{24}}{\left[k_{24}\left(k_{5}+k_{13}\right)+k_{23}\right]^{2}}}\right) .
$$

$\lambda_{\mathrm{CP} \text {-2alt-1 }}$ and $\lambda_{\mathrm{CP} \text {-2alt-2 }}$ are identical to $\lambda_{\mathrm{CP} \text {-2-1 }}$ and $\lambda_{\mathrm{CP} \text {-2-2 }}$ of the GMSK model. Therefore $\lambda_{\text {CP.2alt-1 }}$ is positive and $\lambda_{\text {CP.2alt-2 }}$ neg-

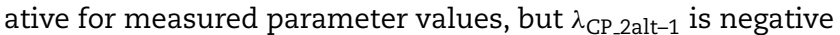
when:

$k_{19}>k_{4}\left(1-k_{20}\right)=1.111$,

while $\lambda_{\mathrm{CP} 2 \text { 2alt-2 }}$ is positive when:

$k_{10}<\frac{k_{1}\left(1-k_{11}\right)}{1+k_{2}}=0.251$.

$\lambda_{\text {CP.5alt-3,4 }}$, however, are always negative, therefore CP $2_{\text {alt }}$ will ${ }_{1148}$ be a saddle point for measured parameter values but an ${ }_{1149}$ asymptotically stable node when $k_{19}>1.111$. However, as $k_{5} 1150$ increases, $P_{2}^{* *}$ reduces, and Eq. (105) reveals CP 2 alt becomes 1151 ecologically infeasible when:

$k_{5}=\frac{k_{23}}{1+k_{24}}=0.799$

Therefore, CP 2 alt leaves the feasible state space well before the mortality parameters reach the crucial value of $1.111 \mathrm{after}$ which previously only $P$ existed. We note therefore that the inclusion of an explicit $P$ mortality term has substantially altered the bifurcation properties of the model.

The location of the critical point (CP $1_{\text {alt }}$ ) at the origin of the system where no biota are extant is unchanged by the addition .

\footnotetext{
135
} 6 7 . . . . . (n) 52 
$1162 \quad N_{1}^{* *}=1$

$1163 \quad B_{1}^{* *}, F_{1}^{* *}, P_{1}^{* *}, Z_{1}^{* *}=0$

$1165 \quad \lambda_{\mathrm{CP} \_1 \text { alt }-1}=\frac{k_{23}}{1+k_{24}}-k_{5}$

$1166 \quad \lambda_{\text {CP_1alt-2 }}=\frac{k_{25}\left(1-k_{11}\right)}{1+k_{26}}-k_{10}$,

$1167 \quad \lambda_{\text {CP_1 } 1 \text { alt-3 }}=-k_{19}$,

$1168 \lambda_{\text {CP_1alt }-4}=-k_{13}$.

1169

1170

1171

1172

1173

$1174 \quad k_{10}>\frac{k_{25}\left(1-k_{11}\right)}{1+k_{26}}=0.397$.

$1175 \lambda_{\text {CP_4alt-1 }}$ is also positive for measured parameter values but is 1176 negative for:

$1177 \quad k_{5}>\frac{k_{23}}{1+k_{24}}=0.799$.

CP $1_{\text {alt }}$ will therefore become an asymptotically stable node when $k_{5}=0.799$. In a climate change scenario where all mortality parameters increase uniformly CP 2 will collide with CP 1 in a transcritical bifurcation when $k_{5}=0.799$, at which point $C P$ 2 becomes infeasible. CP 1 then becomes the long-term state of the system for high mortality rates. There is therefore a fundamental difference between the climate change responses of the GMSK and amended GMSK models: the former predicts an autotroph-dominated ecosystem, while the latter predicts the extinction of all life.

The amended GMSK model also has a more realistic stability criterion for the critical point $\mathrm{CP} 1$, where all living components of the system have died. Eqs. (116)-(119) indicate that the stability of this point is controlled by the mortality terms of the four biotic components. Eqs. (118) and (119) reveal that while the heterotrophs, the zooflagellates $(F)$ and zooplankton (Z), have non-zero mortality CP 1 is potentially stable. The stability of this point is then determined by the growth/mortality ratios of the autotrophs, the bacteria (B) and the phytoplankton $(P)$. While either of these has a growth rate that exceeds its mortality rate, CP 1 will be unstable and life will persist. Note that the stability of CP 1 is not affected by grazing parameters, suggesting that dynamics induced by grazing behaviours are self-regulating and will not result in the extinction of life in the absence external forces.

This result suggests that great care must be taken in selecting ecological models used to predict the impacts of events such as climate change that affect the attributes of organisms represented in the models. The veracity of an ecosystem model is often assessed by testing whether it can reproduce observed data (Franks, 2002). Clearly, the GMSK model can reproduce observed data (Gabric et al., 2003; Cropp et al., 2004), and the modified GMSK model is capable of reproducing the same data, albeit with slightly modified parameter values. However, these models would produce very different results if used to simulate the effects of global warming that pushed their constituent organisms to the limits of their respective ecological niches.

\section{Conclusions}

The GMSK model has a complicated, interesting and quite robust limit cycle that essentially comprises two modes of behaviour that we have called Mode A and Mode B. The robustness of this limit cycle is of profound importance to the model's application in predicting the impacts of climate change and the potential for marine ecosystems to ameliorate that change by the production of DMS. This is because DMS is produced in Mode A and consumed in Mode B.

We found that the switching between Modes A and B was controlled by the coefficients of two linear mortality terms of bacteria and zooflagellates, $k_{10}$ and $k_{13}$. These had similar (but inverse) effects on the timing of the switching between modes, but had very different influences on solution state bifurcations, and the ultimate state of the model for large mortality parameter perturbations. We also found that the other linear mortality term in the model that of zooplankton, $k_{19}$, had quite different effects on the switching behaviour. Cropp et al. (2004) noted that this was an important term for dimethylsulphide production, and hence potential amelioration of climate change, but for different reasons.

The GMSK model may be decomposed into an NPZ submodel and a BFN sub-model. Our analysis has demonstrated that the GMSK model may be usefully described by the sum of its two component sub-models. The dynamics of each of these sub-models combine to form the GMSK model's limit cycle. The shape of this limit cycle is largely determined by the location and stability properties of the critical points. The critical points of the sub-models contribute most of the critical points of the full model, and although the GMSK model has two additional critical points in its own right, the dynamics of the full model appear to be controlled by the critical points of the sub-models. Further, the bifurcations observed in the sub-models are also evident in the full model, although the behaviour in the full model is not as "clean" as observed in the sub-models. This suggests that insight into the behaviour of complicated ecosystem models might be assisted by analysis of any smaller sub-models into which they may reasonably be decomposed.

The NPZ and BFN sub-models add an interesting dimension to the discussion in recent ecological literature on the role of closure terms in influencing the dynamics of ecological models (Edwards and Yool, 2000). Although the NPZ and BFN sub-models both have linear closure terms they have fundamentally different dynamics and bifurcation behaviours. The NPZ and BFN sub-models lend credence to Fulton et al.'s (2003) contention that model dynamics are more strongly influenced by the form of the grazing term than the form of the mortality 
term. However we do note that the coefficients of linear mortality terms and linear closure terms are important bifurcation parameters.

Our results also point to the importance of bacteria in the dynamics of ecosystem models. The presence of bacteria destabilises the plankton model, and bacterial blooms are observed to occur more frequently if bacterial mortality is low or if nutrient levels are high. The presence of a bacterial predator however effectively controls the bacterial population, and restricts the potential for microbial systems to dominate to a small region of parameter space.

We have also highlighted the importance of subtle variations in model formulation if the model is to be used in scenarios such as climate change that dictate that parameter values must change. The inclusion of an explicit linear $P$ mortality term to the GMSK model produces only subtle modifications to the dynamics for measured parameter values. Murray and Parslow (1999) have noted the importance of the formulation of the $P$ mortality term in simple NP models, noting that in the absence of explicit $Z$ representation $P$ mortality is a surrogate of the $\mathrm{Z}$ mortality of NPZ models. Although Murray and Parslow used their models to investigate the effects of nutrient load on coastal ecosystems, they noted that the form of the $P$ mortality term could also determine the stability properties of steady state solutions. While Murray and Parslow considered some $P$ mortality formulations to be inconsistent with observed ecosystem dynamics, the inclusion a linear $P$ mortality term to the GMSK model does not affect the model's ability to reproduce observed ecosystem dynamics, but may have a profound impact on the model's behaviour when it is used in a climate change scenario.

Thus our original and amended models are virtually indistinguishable for our presently observed (experimental) parameter values, but when the climate changes, these models predict totally different outcomes. Our original model predicts a cycling or static autotroph-dominated outcome, whereas the amended model predicts a global extinction. In terms of DMS production, which feeds back on climate change, these two outcomes are profoundly different: the first autotrophdominated outcome predicts a continuous, strong negative feedback on global warming, whereas the second outcome predicts the extinction of life and a run-away greenhouse effect. These very similar models therefore provide explicit examples where the choice of ecological models for inclusion in climate change simulations could dramatically affect the simulation results.

\section{Appendix A. The Jacobian of the NPZ sub-model}

The Jacobian of the NPZ sub-model, after reducing it to an NP system by using the closure of mass condition $N+P+Z=1$ (but retaining $Z$ to simplify the notation) is:

\section{Appendix B. The Jacobian of the BFN sub-model}

The Jacobian of the BFN sub-model, after reducing it to a BF ${ }_{1313}$ system by using the closure of mass condition $B+F+N=1$ (but $\quad 1314$ retaining $N$ to simplify the notation) is:

$J=\left[\begin{array}{ll}\sigma & \varsigma \\ \tau & v\end{array}\right]$,

where

$$
\begin{aligned}
\sigma= & k_{25}\left(1-k_{11}\right)\left(\frac{N^{*}}{N^{*}+k_{26}}\right)-k_{10}-k_{25}\left(1-k_{11}\right) \\
& \times\left(\frac{k_{26}}{\left(N^{*}+k_{26}\right)^{2}}\right) B^{*}-k_{8}\left(\frac{k_{9}}{\left(B^{*}+k_{9}\right)^{2}}\right) F^{*}, \\
\varsigma= & -k_{25}\left(1-k_{11}\right)\left(\frac{k_{26}}{\left(N^{*}+k_{26}\right)^{2}}\right) B^{*}-k_{8}\left(\frac{B^{*}}{B^{*}+k_{9}}\right), \\
\tau= & k_{8}\left(1-k_{14}\right)\left(\frac{k_{9}}{\left(B^{*}+k_{9}\right)^{2}}\right) F^{*}, \\
v= & k_{8}\left(1-k_{14}\right)\left(\frac{B^{*}}{B^{*}+k_{9}}\right)-k_{13} .
\end{aligned}
$$

\section{Appendix C. The Jacobian of the GMSK model}

The Jacobian of the GMSK model, after reducing it to 1324 a BFPZ system by using the closure of mass condition 1325 $B+F+N+P+Z=1$ (but retaining $N$ to simplify the notation) is 1326 given by:

$J=\left[\begin{array}{llll}\alpha & \beta & \chi & \delta \\ \varepsilon & \phi & \varphi & \gamma \\ \eta & 0 & \kappa & 0 \\ \nu & \pi & \varpi & \theta\end{array}\right]$,

where

$\alpha=k_{23}\left(\frac{N^{*}}{N^{*}+k_{24}}\right)-k_{1}\left(\frac{k_{2}}{\left(P^{*}+k_{2}\right)^{2}}\right) B^{*}-k_{4} Z^{*}$

$\beta=-k_{1}\left(\frac{P^{*}}{P^{*}+k_{2}}\right)$,

$\chi=-k_{4} P^{*}$,

$\delta=k_{23}\left(\frac{k_{24}}{\left(\mathrm{P}^{*}+k_{24}\right)^{2}}\right) \mathrm{P}^{*}$, 


$$
\begin{aligned}
1334 \quad \varepsilon= & k_{1}\left(1-k_{11}\right)\left(\frac{k_{2}}{\left(P^{*}+k_{2}\right)^{2}}\right) B^{*}+k_{8}\left(\frac{B^{*}}{B^{*}+k_{9}}\right), \\
1335 & \\
1336 \quad \phi= & k_{1}\left(1-k_{11}\right)\left(\frac{P^{*}}{P^{*}+k_{2}}\right)+k_{25}\left(1-k_{11}\right)\left(\frac{N^{*}}{N^{*}+k_{26}}\right) \\
& -k_{8}\left(\frac{k_{9}}{\left(B^{*}+k_{9}\right)^{2}}\right) F^{*}+k_{8}\left(\frac{B^{*}}{B^{*}+k_{9}}\right)-k_{10}, \\
13378 & \\
1339= & k_{8}\left(\frac{B^{*}}{B^{*}+k_{9}}\right), \\
1340 \quad \gamma= & k_{25}\left(1-k_{11}\right)\left(\frac{k_{26}}{\left(N^{*}+k_{26}\right)^{2}}\right) B^{*}+k_{8}\left(\frac{B^{*}}{B^{*}+k_{9}}\right), \\
1341 \quad \eta= & k_{4}\left(1-k_{20}\right) Z^{*}, \\
1342 \quad \kappa= & k_{4}\left(1-k_{20}\right) P^{*}-k_{19}, \\
1343 \quad & -k_{13}+k_{4} k_{20} Z^{*}, \\
1344 \quad v= & k_{1} k_{11}\left(\frac{k_{2}}{\left(P^{*}+k_{2}\right)^{2}}\right) B^{*}-k_{8} k_{14}\left(\frac{B^{*}}{B^{*}+k_{9}}\right)-k_{23}\left(\frac{N^{*}}{N^{*}+k_{24}}\right) \\
1345 \quad &
\end{aligned}
$$$$
1347 \pi=k_{10}-k_{13}+k_{8} k_{14}\left(\frac{k_{9}}{\left(B^{*}+k_{9}\right)^{2}}\right) F^{*}+k_{1} k_{11}\left(\frac{P^{*}}{P^{*}+k_{2}}\right) B^{*}
$$

\section{REFERENCES}

$$
-k_{8} k_{14}\left(\frac{B^{*}}{B^{*}+k_{9}}\right)-k_{25}\left(1-k_{11}\right)\left(\frac{N^{*}}{N^{*}+k_{24}}\right) \text {, }
$$

$$
\varpi=k_{19}-k_{13}-k_{8} k_{14}\left(\frac{B^{*}}{B^{*}+k_{9}}\right)+k_{4} k_{20} P^{*},
$$

$$
\theta=-k_{13}-k_{25}\left(1-k_{11}\right)\left(\frac{k_{26}}{\left(N^{*}+k_{26}\right)^{2}}\right) B^{*}-k_{8} k_{14}\left(\frac{B^{*}}{B^{*}+k_{9}}\right)
$$

$$
-k_{23}\left(\frac{k_{24}}{\left(N^{*}+k_{24}\right)^{2}}\right) P^{*} \text {. }
$$

Bazykin, A.D., 1998. Nonlinear Dynamics of Interacting Populations. World Scientific, Singapore, 193 pp.
Boyce, W.E., DiPrima, R.C., 1997. Elementary Differential Equations and Boundary Value Problems. John Wiley and Sons Inc., New York, 749 pp.

Charlson, R.J., Lovelock, J.E., Andreae, M.O., Warren, S.G., 1987. Oceanic phytoplankton, atmospheric sulphur, cloud albedo and climate. Nature 326, 655-661.

Cropp, R.A., Norbury, J., Gabric, A., Braddock, R., 2004. Modeling dimethylsulphide production in the upper ocean. Global Biogeochem. Cycles 18, doi:10.1029/2003GB002126.

DeAngelis, D.L., 1980. Energy flow, nutrient cycling and ecosystem resilience. Ecology 61, 764-771.

Edwards, A.M., Bees, M.A., 2001. Generic dynamics of a simple plankton population model with a non-integer exponent of closure. Chaos Solitons Fractals 12, 289-300.

Edwards, A.M., Brindley, J., 1996. Oscillatory behaviour in a three component plankton population model. Dyn. Stab. Syst. 11, 347-370.

Edwards, A.M., Brindley, J., 1999. Zooplankton mortality and the dynamical behaviour of plankton population models. Bull. Math. Biol. 61, 303-339.

Edwards, A.M., Yool, A., 2000. The role of higher predation in plankton population models. J. Plankton Res. 22, 10851112.

Franks, P.J.S., 2002. NPZ models of plankton dynamics: their construction, coupling to physics, and application. J. Oceanogr. 58, 379-387.

Fulton, E.A., Smith, A.D.M., Johnson, C.R., 2003. Mortality and predation in ecosystem models: is it important how these are expressed? Ecol. Model. 169, 157-178.

Gabric, A.J., Cropp, R.A., Hirst, A.C., Marchant, H.J., 2003. The response of dimethylsulphide production to simulated warming in the eastern Antarctic Southern Ocean. Tellus 55B, 966-981.

Gabric, A.J., Matrai, P.A., Vernet, M., 1999. Modelling the production and cycling of dimethylsulphide during the vernal bloom in the Barents Sea. Tellus 51B, 919-937.

Gabric, A.J., Murray, N., Stone, L., Kohl, M., 1993. Modeling the production of dimethylsulfide during a phytoplankton bloom. J. Geophys. Res. 98, 22,805-22,816.

Gabric, A.J., Simo, R., Cropp, R.A., Hirst, A.C., Dachs, J., 2004. Global estimates of the oceanic emission of dimethylsulfide under enhanced greenhouse conditions. Global Biogeochem. Cycles 18, doi:10.1029/2003GB002183.

Moloney, C.L., Bergh, M.O., Field, J.G., Newell, R.C., 1986. The effect of sedimentation and microbial nitrogen regeneration in a plankton community: a simulation investigation. J. Plankton Res. 8, 427-445.

Murray, A.G., Parslow, J.S., 1999. The analysis of alternative formulations in a simple model of a coastal ecosystem. Ecol. Model. 119, 149-166.

Steele, J.H., Henderson, E.W., 1992. The role of predation in plankton models. J. Plankton Res. 14, 157-172.

Wolfe, A., Swift, J.B., Swinney, H.L., Vastano, J.A., 1985. Determining Lyapunov exponents from a time series. Physica D 16, 285-317. 